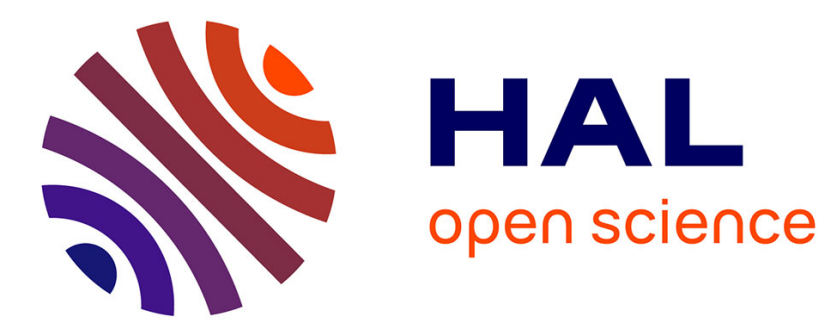

\title{
On the linear receptivity of trailing vortices
}

Tobias Bölle, Vincent Brion, Jean-Christophe Robinet, Denis Sipp, Laurent

Jacquin

\section{To cite this version:}

Tobias Bölle, Vincent Brion, Jean-Christophe Robinet, Denis Sipp, Laurent Jacquin. On the linear receptivity of trailing vortices. Journal of Fluid Mechanics, 2020, 908, pp.29. 10.1017/jfm.2020.898 . hal-03048005

\section{HAL Id: hal-03048005 \\ https://hal.science/hal-03048005}

Submitted on 9 Dec 2020

HAL is a multi-disciplinary open access archive for the deposit and dissemination of scientific research documents, whether they are published or not. The documents may come from teaching and research institutions in France or abroad, or from public or private research centers.
L'archive ouverte pluridisciplinaire HAL, est destinée au dépôt et à la diffusion de documents scientifiques de niveau recherche, publiés ou non, émanant des établissements d'enseignement et de recherche français ou étrangers, des laboratoires publics ou privés. 


\title{
On the linear receptivity of trailing vortices
}

\author{
Tobias Bölle $^{1,} \dagger$, Vincent Brion ${ }^{1}$, Jean-Christophe Robinet ${ }^{2}$, Denis Sipp ${ }^{1}$ \\ and Laurent Jacquin ${ }^{1}$ \\ ${ }^{1}$ DAAA, ONERA-The French Aerospace Lab, Université Paris Saclay, 92190 Meudon, France \\ ${ }^{2}$ DynFLuid, Arts et Métiers Institute of Technology, CNAM, HESAM University, 75013 Paris, France
}

(Received 24 March 2020; revised 28 July 2020; accepted 2 October 2020)

\begin{abstract}
The present work investigates the excitation process by which free-stream disturbances are transformed into vortex-core perturbations. This problem of receptivity is modelled in terms of the resolvent in frequency space as the linear response to forcing. This formulation of receptivity suggests that non-normality of the resolvent is necessary to allow free-stream disturbances to excite the vortex core. Considering a local (in frequency) measure of non-normality, we show that vortices are frequency-selectively non-normal in a narrow frequency band of retrograde perturbations while the rest of the range is governed by an effectively normal operator, thus not contributing to receptivity. Canonical decomposition of the resolvent reveals that vortices are most susceptible to coiled filaments localised about the critical layer that induce bending waves on the core. Considering Lamb-Oseen, Batchelor and Moore-Saffman vortices as reference-flow models, we find free-stream receptivity to be essentially generic and independent of the axial wavelength on the considered range. A stochastic interpretation of the results could be a model for trailing-vortex meandering.
\end{abstract}

Key words: vortex dynamics, general fluid mechanics

\section{Introduction}

Trailing-vortex experiments systematically display an unsteady dynamics manifesting as the lateral displacement of the vortex, called meandering. Despite having a broadband spectral signature, kinetic energy is typically essentially concentrated in a low-frequency bending wave (Devenport et al. 1996; Jacquin et al. 2001; Bailey et al. 2018). Similar dynamics is observed for related configurations such as inlet vortices or tornadoes (Wang \& Gursul 2012; Karami et al. 2019) as well as in the interaction of canonical vortices with turbulence (Melander \& Hussain 1993; Marshall \& Beninati 2005). For this reason, we only consider helical perturbations with $|m|=1$ ( $m$ is the azimuthal wavenumber).

Discussion of the origin of vortex unsteadiness divides researchers into two camps, who attribute it either to intrinsic or extrinsic mechanisms. The former approach assumes that the dynamics is governed in essence by the vortex in isolation, e.g. instability or self-induction (Fabre \& Jacquin 2004; Ting, Klein \& Knio 2007). We assume the second approach, assessing vortex unsteadiness as a consequence of external disturbances (e.g. residual turbulence in wind tunnels or the atmosphere or residual vortex sheets from the

$\dagger$ Email address for correspondence: tobias.bolle@onera.fr 
roll-up of the vortex itself). The excitation of vortex-core perturbations by the surrounding free stream is a (generalised) receptivity problem.

There is no reason to believe that receptivity in general should be a linear mechanism (Saric, Reed \& Kerschen 2002) and nonlinear studies have been pursued. Nevertheless, trailing-vortex experiments provide evidence that variation of the free-stream turbulence intensity only affects the displacement amplitude linearly (Van Jaarsveld et al. 2011, p. 222) while the dominant meandering frequency is universal irrespective of the turbulence intensity (Bailey et al. 2018, figure 7). These findings altogether suggest that vortices tend to spatially separate dynamical regimes which interact linearly (at least for the question of meandering). That is, on the one hand the free-stream dynamics is nonlinear and complex whereas on the other hand the vortex response is governed by a core dynamics of organised nature (Bandyopadhyay, Stead \& Ash 1991; Devenport et al. 1996; Jacquin et al. 2001). This distinction is generic and independent of the (structural) details of free-stream turbulence (at least sufficiently far from the wing, approximately five chord lengths, say). For this reason, we neglect all internal structure and interactions of the advective nonlinearity $\left(\sim \nabla_{u} u, u\right.$ being the perturbation velocity about the reference state $U$ ) but simply consider its compiled action in terms of the forcing field $f=f(\boldsymbol{u})$ (Kato \& Fujita 1962, p. 244; Landahl 1967, p. 456; Sharma, Mezić \& McKeon 2016, p. 6). Identification of the free stream with the given forcing field $f(t)$, means that the exact evolution equation for the perturbations $\boldsymbol{u}(t)$ (cf. Joseph 1976, p. 8) takes on the form of an inhomogeneous linear abstract Cauchy problem. The general solution in the time domain is the sum of the homogeneous $\boldsymbol{u}_{h}(t)$ and a particular solution $\boldsymbol{u}_{p}(t)$. By Duhamel's principle,

$$
\boldsymbol{u}(t)=\boldsymbol{u}_{h}(t)+\boldsymbol{u}_{p}(t)=T(t) \boldsymbol{u}(0)+\int_{0}^{t} \mathrm{~d} \tau T(t-\tau) \boldsymbol{f}(\tau), \quad t \geq 0,
$$

where $t \mapsto T(t)$ is the propagator (semigroup) generated by the linearised Navier-Stokes operator L (Kato 1980, p. 488; Engel \& Nagel 2000, p. 436; see also Kato \& Fujita 1962, pp. 244-245; Sohr 2001, p. 8).

Alternatively, the solution in frequency space is obtained by Laplace transform of (1.1),

$$
\hat{\boldsymbol{u}}(s)=R(s)(\boldsymbol{u}(0)+\hat{\boldsymbol{f}}(s)) \text { for admissible frequency } s \in \mathbb{C} .
$$

The family of transfer operators $s \mapsto R(s)=\int_{0}^{\infty} \mathrm{d} t \exp (-s t) T(t)$ is called the resolvent (Kato 1980, p. 484; Engel \& Nagel 2000, p. 58). Equation (1.2) shows that the resolvent determines the homogeneous and particular solution in frequency space. The steady-state response of an asymptotically stable system (the case here) corresponds to the particular solution in (1.1) and is characterised in frequency space by taking the Fourier transform. Roughly speaking, setting $s=\mathrm{i} \omega, \omega \in \mathbb{R}$ in (1.2) and $\boldsymbol{u}(0)=0$ we study the response to harmonic forcing.

So far, most analysis of linear vortex dynamics has concentrated on the representation in the time domain. The homogeneous solution of (1.1) governs the transient dynamics for all $t \geq 0$ while asymptotic stability (as $t \rightarrow \infty$ ) is determined by the spectrum of $L$ (Joseph 1976, p. 9; Arnol'd 1992, p. 212). Linear stability of Lamb-Oseen, Batchelor and Moore-Saffman vortices has been studied by Fabre, Sipp \& Jacquin (2006), Fabre \& Jacquin (2004) and Feys \& Maslowe (2014) among others. From these studies, one concludes that vortices are asymptotically stable for typical parameters of aeronautical applications. More importantly, the spectrum is the union of a discrete spectrum of isolated eigenvalues (governing asymptotic stability) and two continuous spectra due to spatial 
unboundedness and an inviscid singularity, respectively (strictly speaking, continuity of the latter is true only in the inviscid limit; Heaton \& Peake 2007). It is in fact this latter inviscid continuous spectrum which seems to be of primary importance for the transient dynamics (Heaton \& Peake 2007, p. 294; Mao \& Sherwin 2012, p. 42).

As a matter of fact, despite asymptotic stability, transient energy growth is possible if the linear operator $L$ is non-normal (i.e. it does not commute with its adjoint) (Farrell \& Ioannou 1996; Trefethen \& Embree 2005, § 14). Canonical decomposition (as in §3.2) of the propagator $T(t)$ for fixed $t>0$ identifies those pairs of optimal initial and associated final-time perturbations which maximise energy growth for the given $t$. The possibility of transient growth has been shown numerically for Lamb-Oseen and Batchelor vortices by Antkowiak \& Brancher (2004), Pradeep \& Hussain (2006), Heaton \& Peake (2007) and Mao \& Sherwin (2012) among others. For helical perturbations with $|m|=1$, the main finding is the resonant excitation of core bending waves by remote filaments in the core periphery (Antkowiak \& Brancher 2004). This mechanism also applies for the Batchelor vortex (Heaton \& Peake 2007, p. 295).

Stochastic-forcing analysis in the time domain is concerned with the particular solution of (1.1) assuming a stationary dynamics, viz. considering the $t \rightarrow \infty$ limit (Farrell \& Ioannou 1996). The probability distribution of $f(t)$ is a priori unknown and the forcing is usually assumed to be Gaussian spatio-temporal white noise (Farrell \& Ioannou 1996; Fontane, Brancher \& Fabre 2008; Towne, Schmidt \& Colonius 2018). Stochastic-forcing analysis has been conducted by Fontane et al. (2008) and Guo, Chen \& Sun (2011) for the Lamb-Oseen and Batchelor vortices, respectively. These studies identify the same resonance prototype as in transient-growth analyses to contribute most to stationary kinetic perturbation energy and covariance.

Fewer studies dealt with the solution in frequency space (1.2). Instead of analysing the propagator, the transient dynamics can be described from tracing out contours of the resolvent norm, i.e. the pseudospectrum, in the complex plane (Trefethen \& Embree 2005, pp. 31-135). To the best of our knowledge, pseudospectra have only been computed for axisymmetric $(m=0)$ perturbations of the Batchelor vortex (Mao \& Sherwin 2011). We compute the pseudospectrum of the Lamb-Oseen vortex for $m=1$ and show that it is qualitatively identical to the results of Mao \& Sherwin (2011) despite the important parametric difference.

However, by appeal to the above motivated physical model of trailing-vortex dynamics, the majority of our analysis concerns response to harmonic forcing (i.e. for $s=\mathrm{i} \omega, \omega \in \mathbb{R}$ in $(1.2)$ and $\boldsymbol{u}(0)=0)$. As in transient-growth studies, canonical decomposition $(\S 3.2)$ of the resolvent $R(\mathrm{i} \omega)$ for fixed $\omega \in \mathbb{R}$ yields pairs of spatial forcing and response structures which are optimal in terms of energy amplification. Canonical decomposition of the resolvent has been computed previously for Lamb-Oseen and Batchelor vortices by Guo \& Sun (2011) and Blanco-Rodríguez et al. (2017) for $|m| \in\{0,1,2\}$. However, no thorough attempt has been made to relate these results to physics and linear dynamics in general. Recently, Viola, Arratia \& Gallaire (2016) analysed the resolvent of perturbation dynamics about the non-parallel, convectively unstable Batchelor vortex in a global approach and compared their results to linear stability analysis and nonlinear simulations of the Navier-Stokes equations. Their work focused on mode selection (i.e. identification of dominant azimuthal wavenumbers) and concludes applicability of the resolvent for this question.

The resolvent for harmonic forcing is a convenient model for linear receptivity to sustained excitation by the free stream (cf. also McKeon \& Sharma 2010, p. 342). Assuming the forcing to be the compiled action of the (turbulent) free stream that we have no further knowledge of, $f(t)$ is naturally modelled as a random process. In this case the 
response $\boldsymbol{u}(t)$ is also a stochastic process $(T(t), R(s)$ are deterministic) and $\boldsymbol{U}=$ const. is the mean state. It should be noted that $\boldsymbol{U}+\boldsymbol{u}(t)$ is the Reynolds decomposition where the perturbation may be finite (Towne et al. 2018, p. 836). This setting is to be contrasted with traditional linear analysis where $U$ is the base flow (i.e. a fixed point of the Navier-Stokes equations; Arnol'd 1992, p. 210) and $\boldsymbol{u}(t)$ is an infinitesimal deterministic process. Despite the motivation, the mathematical framework we employ does not distinguish between deterministic or stochastic forcing, neither is the restriction to free-stream disturbances essential and $f(t)$ could conceptually represent e.g. control. Our analysis only addresses the operator properties of the (deterministic) resolvent, specifically its non-normality.

The notion of free-stream receptivity crucially relies on remote excitation in the sense that forcing and response have spatially distinct support. Non-normality of the resolvent is a means to excite core perturbations by radially disjoint forcing structures (cf. also Trefethen \& Embree 2005, p. 201). We conclude that non-normality is necessary for linear free-stream receptivity (conjecture $3.1, \S 2$ ). Since non-normality is the essential feature of linear vortex dynamics in general, we expect our results to be of fundamental interest beyond the particular question of free-stream receptivity.

Against this backdrop, the main objective must be a thorough understanding and quantification of the resolvent non-normality. Various scalar measures have been proposed (cf. Trefethen \& Embree 2005, § 48) which, however, might significantly overestimate the effective non-normality and hence be misleading for actual dynamics. Trefethen \& Embree (2005, p. 446) conclude that "nonnormality is too complex to be summarised in a single number'. We make use of resolvent bounds to classify non-normality of the linear vortex dynamics in the frequency domain. While the governing operator is non-normal, it will be shown that the effective behaviour for vortices depends on the excitation frequency, we call this selective non-normality (conjecture 4.1, $\S 3$ and 4). First studying the linearised operator $L$ for a generic vortex in $\S 4.1$, we deduce that non-normality should be maximal for forcing localised about the critical layer (radial location where the phase velocity equals advection by the mean; Le Dizès 2004, p. 319). Assuming the (parallel) Lamb-Oseen vortex as a reference state (base-flow model), we confirm this result numerically in $\S \S 4.2$ and 4.4 .

The second objective is the corroboration of these results under variation of the reference flow and the axial wavenumber. For this purpose, we analyse the resolvent for (parallel) Batchelor and Moore-Saffman vortices in $\S 5$ which constitute approximations to experimental mean flow (see $\S 2.2$ ). This sequence of reference states serves two purposes, namely to assess differences between the dynamics about base and mean flows as motivated above and to evaluate the impact of (weak) axial mean velocity in the vortex core. We discuss the relation to previous linear studies throughout.

Lastly, results in frequency space are often easier related to experiments than time-domain analysis. Still, idealising vortex meandering as a monochromatic wave, the associated frequency in experiments corresponds to an infinity of wavenumber-frequency pairs in theory by the Doppler relation. Nevertheless, by appeal to our results of selective non-normality, in $\S 6$ we show how the actually contributing frequency range can be significantly reduced.

\section{Linear receptivity in the resolvent formalism}

Let the fluid domain be the entire Euclidean space $\mathbb{R}^{3}$ with cylindrical coordinates $\boldsymbol{x}=(r, \theta, z)$ and corresponding velocities $\boldsymbol{v}=\left(v_{r}, v_{\theta}, v_{z}\right)$. The $z$ coordinate is chosen to coincide with the axis of mean rotation, $r$ pointing radially outwards and $\theta$ being such that 
$\{r, \theta, z\}$ yields a right-handed system. The fluid is assumed incompressible with constant, homogeneous material properties.

All physical quantities are non-dimensionalised on the length scale $r_{0}=2 \sqrt{v t_{0}}$, where $t_{0}>0$ determines the vortex age and is chosen such that $r_{0}:=1$ without loss of generality, and the azimuthal mean velocity at this radius $\left(2 \pi r_{0}\right)^{-1} \Gamma_{0}:=1$. It follows the circulation-based Reynolds number $R_{\Gamma}:=\Gamma_{0} /(2 \pi \nu)=v^{-1}$ ( $v$ is the kinematic viscosity).

\subsection{Trailing-vortex dynamics as a generalised receptivity problem}

The dynamics of trailing vortices in experiments is dominated by low-frequency displacement waves (vortex meandering). In the intermediate wake $(z \leq 10 b, b$ is the wingspan; Jacquin et al. 2001, p. 5) destabilising effects from consideration of the counter-rotating pair are of second order (Crow $\sim b^{-2}$; Jacquin et al. 2001, p. 17) and the isolated line vortex is asymptotically stable for parameters of typical aeronautic applications (Fabre \& Jacquin 2004, p. 259). Rather, it appears that the observed dynamics is due to temporally sustained excitation of the vortex by the surrounding free stream (as already suggested by Baker et al. 1974, p. 331). This internalisation of external disturbances is reminiscent of receptivity.

While strictly speaking the classical notion of receptivity applies to the transition problem and the excitation of instability modes (Morkovin 1988, p. 76), it is used here to describe the general reaction of a system to initial or temporally sustained external disturbances (see also Fontane et al. 2008, p. 236). For these reasons, let us refer to the problem of receptivity in the following generalised sense which is not restricted to laminar reference states but straightforwardly extends to turbulent mean flows. (This generalised perception of receptivity is also implicitly understood in McKeon \& Sharma 2010, p. 342 and Towne et al. 2018, §5.1 among others.)

DEFINITION 2.1. The excitation of general perturbations inside the vortex by external disturbances is called (free-stream) receptivity.

The essential aspect of receptivity according to definition 2.1 is perturbation internalisation in the sense that external disturbances in the free stream are converted into internal perturbations inside the vortex. For this excitation to be well defined, we must partition the fluid domain into a subset $\mathbb{V}$ identified with the interior of the vortex and its complement $\mathbb{R}^{3} \backslash \mathbb{V}$, viz. the free stream.

Trailing-vortex experiments provide considerable evidence that rather rapidly (within approximately two wing chords $c$ at chord-based Reynolds number $R_{c}:=U_{\infty} c / v \sim 10^{5}$; Devenport et al. 1996, p. 68) the flow develops a coherent vortex in the sense of a single concentration of streamwise vorticity which is axisymmetric and parallel to leading order. For definiteness, we define the system boundary as a concentric cylinder at the vortex-core radius $r_{1}=1.12$, corresponding to the location of the maximum azimuthal velocity of a Gaussian vortex. Similar identification of the vortex core is used by Pradeep \& Hussain (2006, p. 266) and Takahashi, Ishii \& Miyazaki (2005, p. 6) for example. Thus, perturbations with radial support less than $r_{1}$ are interior to the vortex while those disturbances supported on $r>r_{1}$ are external, viz. in the free stream. It must be stressed that the notion of internalisation here only serves the purpose to highlight the essential aspect of receptivity (according to definition 2.1) of being inherently related to a spatial shift between forcing and response in the (spatio-temporal) fluid domain. In fact, as discussed in $\$ 3.1$, free-stream receptivity may not require actual transport (e.g. of energy) over the system boundary. 
Trailing vortices are generically associated with an axisymmetric mean velocity of the form $U(r)=U_{\theta}(r) \boldsymbol{e}_{\theta}+U_{z}(r) \boldsymbol{e}_{z}$, blending rotational and jet kinematics. Restriction to parallel vortices is justified by previous studies of Antkowiak (2005, figure 3.18), Heaton, Nichols \& Schmid (2009) and Viola et al. (2016, figure 5), showing numerically that consideration of base-flow diffusion does not alter considerably transient-growth and stability properties.

We consider receptivity of Lamb-Oseen, Batchelor and Moore-Saffman vortices. The motivation for this sequence of reference flows is twofold. Firstly, it gradually shifts between different conceptual points of view, viz. from base to mean flow. Considering the Lamb-Oseen vortex as (an approximation to) a base flow yields receptivity of the laminar state. On the other hand, the Moore-Saffman vortex rather constitutes an approximation to the mean flow, thus building on the above generalised notion of receptivity in a turbulent (stochastic) framework. Secondly, the effect of including an axial mean velocity on free-stream receptivity can be assessed (we assume weak axial mean velocity as discussed below).

The parallel approximation of the Batchelor vortex (Batchelor 1964) reads (see also Fabre \& Jacquin 2004, p. 242 and Heaton \& Peake 2007, p. 285)

$$
U_{r}(r)=0, \quad U_{\theta}(r)=\frac{1-\mathrm{e}^{-r^{2}}}{r}, \quad U_{z}(r)=q^{-1} \mathrm{e}^{-r^{2}} .
$$

Equation $(2.1 a-c)$ is parametrised by the swirl number $q \neq 0$ (the ratio of mean azimuthal to axial velocity; Jacquin et al. 2001, p. 15). The Lamb-Oseen vortex is formally obtained as the $|q| \rightarrow \infty$ limit of the Batchelor vortex $(2.1 a-c)$.

The parallel approximation of the Batchelor vortex is typically considered as a base flow in a stability analysis. Nevertheless, Iungo (2017, p. 1785) observe the Batchelor vortex to fit experimental data well, which is also claimed by Heaton \& Peake (2007, p. 272). Qualitative matching is equally reported in numerical studies of Takahashi et al. (2005, p. 5) and Heaton et al. (2009, pp. 142-144). At least, the Batchelor vortex constitutes a useful prototype, containing the essential aspects of trailing-vortex mean velocity.

The Moore-Saffman vortex is defined by a system of differential equations (Moore \& Saffman 1973) which is solved numerically. From a practical point of view the important aspect of this model is its parametrisation by the real value $n \in(0,1)$, which determines the radial decay of the velocity profiles and leads to jet-wake coexistence for sufficiently small values. Generally, the Moore-Saffman vortex is observed to be a good fit to the experimental mean velocity. There is considerable experimental evidence that a representative value is about $n \gtrsim 0.75$ for trailing vortices. For instance, experiments conducted at ONERA suggest a calibration with $n \in\{0.79,0.72,0.80\}$ in the streamwise range of one to five wingspans (P. Molton, private communication). Similarly, experiments and implicit large eddy simulation (iLES) of García-Ortiz et al. (2019, figure 6b) report a range of roughly $n \in[0.8,0.95]$ over a streamwise range of 40 chords and chord Reynolds number $R_{c} \sim 10^{4}$. For these representative values of $n$ departure from a Gaussian vortex is essentially negligible.

\subsection{Resolvent for the linear dynamics of a trailing vortex}

Let there be given a time-invariant reference state $(\boldsymbol{U}, P)$ of the form $U(r)=U_{\theta}(r) \boldsymbol{e}_{\theta}+$ $U_{z}(r) \boldsymbol{e}_{z}$ and $P=P(r)$ (the pressure) defining the vortex in the sense of $\S 2.1$ by one of the reference states of $\S 2.2$, subjected to the perturbation $(\boldsymbol{u}, p)$ such that $(\boldsymbol{U}+\boldsymbol{u}$, 
$P+p)$ solves the Navier-Stokes equations. Consider perturbations in the form of Fourier modes $\boldsymbol{u}(t, r, \theta, z)=\hat{\boldsymbol{u}}(s, r, m, \alpha) \exp (\mathrm{i}(m \theta+\alpha z)-s t)+$ c.c. whereas $m \in \mathbb{Z}, \alpha \in \mathbb{R}$ and $s=s_{r}+\mathrm{i} s_{i} \in \mathbb{C}$ and equivalently for the pressure. For convenience, parameters in the Fourier amplitudes will be dropped if unambiguous. Complex frequencies $s$ are used in the computation of spectra and pseudospectra (defined below) while the response to temporally sustained forcing assumes purely imaginary values $s=\mathrm{i} \omega, \omega \in \mathbb{R}$. We seek perturbations with finite kinetic energy, thus endowing the solution space with the inner product

$$
(\hat{\boldsymbol{u}}, \hat{\boldsymbol{v}}):=\int_{0}^{\infty} \mathrm{d} r r \sum_{l=1}^{3} \overline{\hat{u}_{l}(r)} \hat{v}_{l}(r),
$$

where an overbar $\overline{(\cdot)}$ denotes complex conjugation.

Inserting the decomposition $(\boldsymbol{U}+\boldsymbol{u}, P+p)$ into the Navier-Stokes equations and subtracting the equation for the reference flow yields a nonlinear transport equation for the perturbation. Restriction only to linear terms yields the linearised perturbation transport equation (Joseph 1976, pp. 7-8). In studying receptivity, we suppose a non-vanishing inhomogeneity $f$ to drive the system. Introducing the above Fourier ansatz into the linear perturbation transport equation yields a boundary-value problem on $r \in[0, \infty)$ for the system of linear ordinary differential equations, parametrised by the wavenumbers $m \in \mathbb{Z}, \alpha \in \mathbb{R}$ and frequency $s \in \mathbb{C}$. For $m= \pm 1$,

$$
(L-s P)\left(\begin{array}{l}
\hat{u} \\
\hat{p}
\end{array}\right)=\left(\begin{array}{l}
\hat{f} \\
0
\end{array}\right) \text { such that }\left\{\begin{array}{l}
\mathrm{d} \hat{u}_{r} /\left.\mathrm{d} r\right|_{0} \text { and } \mathrm{d} \hat{u}_{\theta} /\left.\mathrm{d} r\right|_{0}=0, \\
\hat{u}_{z}(0)=\hat{p}(0)=0,
\end{array} \quad\left(\begin{array}{l}
\hat{u} \\
\hat{p}
\end{array}\right)(r \rightarrow \infty) \rightarrow 0 .\right.
$$

The restriction to perturbations with finite kinetic energy on an unbounded domain requires faster than algebraic decay as $r \rightarrow \infty$ (Ash \& Khorrami 1995, pp. 339-342). The linear operators in (2.3) are formally given by the projection

$$
P:=\left[\begin{array}{llll}
1 & 0 & 0 & 0 \\
0 & 1 & 0 & 0 \\
0 & 0 & 1 & 0 \\
0 & 0 & 0 & 0
\end{array}\right]=\left[\begin{array}{lll}
1 & 0 & 0 \\
0 & 1 & 0 \\
0 & 0 & 1 \\
0 & 0 & 0
\end{array}\right]\left[\begin{array}{llll}
1 & 0 & 0 & 0 \\
0 & 1 & 0 & 0 \\
0 & 0 & 1 & 0
\end{array}\right]=: B B^{\dagger}
$$

and

$L:=\left[\begin{array}{cccc}\mathrm{i} m \Omega+\mathrm{i} \alpha U_{z}-v\left(\Delta-r^{-2}\right) & -2 \Omega+2 v \mathrm{i} m r^{-2} & 0 & \mathrm{~d} / \mathrm{d} r \\ W_{z}-2 v \mathrm{i} m r^{-2} & \mathrm{i} m \Omega+\mathrm{i} \alpha U_{z}-v\left(\Delta-r^{-2}\right) & 0 & \mathrm{i} m r^{-1} \\ \mathrm{~d} U_{z} / \mathrm{d} r & 0 & \mathrm{i} m \Omega+\mathrm{i} \alpha U_{z}-v \Delta & \mathrm{i} \alpha \\ -r^{-1}-\mathrm{d} / \mathrm{d} r & -\mathrm{i} m r^{-1} & -\mathrm{i} \alpha & 0\end{array}\right]$

whereas

$$
\Delta:=\frac{\mathrm{d}^{2}}{\mathrm{~d} r^{2}}+\frac{1}{r} \frac{\mathrm{d}}{\mathrm{dr}}-\left(\frac{m}{r}\right)^{2}-\alpha^{2} \quad \text { and } \quad \Omega:=\frac{U_{\theta}}{r}, \quad W_{z}:=\Omega+\frac{\mathrm{d} U_{\theta}}{\mathrm{d} r}
$$

are the Laplace operator of a scalar field, the angular velocity and axial vorticity of the reference flow, respectively. The radius $r_{c} \in \mathbb{R}$ for which mean advection 
$m \Omega\left(r_{c}\right)+\alpha U_{z}\left(r_{c}\right)$ equals the perturbation frequency $\omega=s_{i} \in \mathbb{R}$ is called critical layer (Le Dizès 2004, p. 319).

Suppose the inverse of (2.3) exists, then the solution formally reads

$$
\hat{\boldsymbol{u}}(s)=B^{\dagger}(L-s P)^{-1} B \hat{f}(s) \text { for all } s \in \rho(L),
$$

and the operator-valued one-parameter family $s \mapsto R(s ; L):=B^{\dagger}(L-s P)^{-1} B$ is referred to as the resolvent (Kato 1980, p. 173). Bounded inversion exists for frequencies which do not pertain to the spectrum $\sigma(L)$. The subset of the complex plane for which the resolvent is defined and bounded is called the resolvent set $\rho(L)$ (Riesz \& Sz.-Nagy $1956, \S 132)$. For the asymptotically stable systems considered here $i \mathbb{R} \subset \rho(L)$ holds and the resolvent is defined on the entire imaginary axis. The pseudospectrum is defined by $\sigma_{\epsilon}(L):=\left\{s \in \mathbb{C} \mid\|R(s ; L)\|>\epsilon^{-1}\right\}$ as contours of the resolvent norm (defined in $\S 3.2$ ) for fixed values of $\epsilon>0$ (Trefethen \& Embree 2005, p. 31).

The resolvent (2.7) is obtained numerically from finite-element discretisation of (2.3) and inversion of the corresponding matrix, see the Appendix.

Due to symmetries of the linearised perturbation (2.3) the parameter space can be reduced. For the Lamb-Oseen vortex it is sufficient to consider $m, \alpha \geq 0$ and $\omega \in \mathbb{R}$ (Fabre et al. 2006, pp. 241-242). Inclusion of an axial velocity component breaks azimuthal symmetry, making a distinction between positive and negative azimuthal wavenumbers necessary (Fabre \& Jacquin 2004, p. 247; Heaton \& Peake 2007, p. 289).

\section{Estimation of linear receptivity by analysis of the non-normality}

Let $A$ be a formal operator on a Hilbert space (see e.g. Kato 1980, p. 146 for this terminology) and denote by $A^{\dagger}$ its formal adjoint. Then $A$ is formally normal if the commutator $[\cdot, \cdot]$ of $A$ and $A^{\dagger}$ is equal to zero, i.e. $\left[A, A^{\dagger}\right]:=A A^{\dagger}-A^{\dagger} A=0$ (Riesz \& Sz.-Nagy 1956, p. 284; Kato 1980, p. 276).

\subsection{Necessity of non-normality for linear free-stream receptivity}

Receptivity according to definition 2.1 is intimately linked to the excitation of vortex-core perturbations by spatially remote disturbances. For receptivity of vortices to free-stream disturbances this implies a radial perturbation shift in order to internalise external disturbances. Now, if the linear receptivity problem (2.7) is associated with a normal operator $R(\mathrm{i} \omega ; L)$ any forcing-response pair $\{\hat{f}(\omega), \hat{\boldsymbol{u}}(\omega)\}$ should have the same radial support. On the other hand, Trefethen \& Embree (2005, p. 10) states that resonance of non-normal systems is the fundamental principle in receptivity. Indeed, the following may be suggested (see also Roy \& Subramanian 2014, p. 405).

CONJECTURE 3.1. Let perturbations have finite kinetic energy, then non-normality of $R(\mathrm{i} \omega ; L)$ is necessary for the linear model of vortex receptivity to free-stream disturbances.

Assuming a linear dynamics, vortices are receptive to free-stream disturbances by two distinct mechanisms, namely through (i) generalised eigenvectors pertaining to the continuous spectrum $\sigma_{c}^{\infty}(L)$ (defined in $\S 4.2$ ) and (ii) critical-layer forcing discussed in $\S \S 4.2$ and 4.3. Efficiency of the former is typically significantly diminished due to shear sheltering such that disturbances only slightly penetrate the core (Jacobs \& Durbin 1998). In fact, the penetration mechanism is viscous (no penetration in the inviscid limit; Jacobs \& Durbin 1998, p. 2010) and should not be significant for high Reynolds numbers 
in experiments. Restriction to perturbations with finite kinetic energy in conjecture 3.1 excludes receptivity associated with generalised eigenvectors pertaining to $\sigma_{c}^{\infty}(L)$.

There is considerable evidence that a vortex essentially constitutes a material subset of the fluid domain which does not exchange fluid particles with its surrounding (Haller et al. 2016). Therefore, perturbation-energy amplification in the core must preclude significant mass or momentum transport, e.g. through intermittent vorticity stripping or ejection, as proposed by Bandyopadhyay et al. (1991, pp. 1629, 1633). Indeed, strong ambient turbulence intensity is required to enable exchange of core fluid with the free stream (Marshall \& Beninati 2005, pp. 231-233). For low to moderate levels, numerical experiments indicate that coiled vorticity filaments in the free stream cannot penetrate into the core (Jacobs \& Durbin 1998, p. 2006; Takahashi et al. 2005, p. 12). Low turbulence intensities in experiments therefore call for receptivity mechanisms which excite core perturbations without significant mass transport. The proposed receptivity mechanism by non-normality does not require physical exchange of fluid, hence, constitutes a candidate in moderate-turbulence regimes.

\subsection{Canonical decomposition and bounds on the resolvent}

The present study uses canonical decomposition of the resolvent, cf. (2.7). Let $R(s ; L)$ be a compact linear operator and $n>0$. Then, for all admissible forcing fields $\hat{f}$, the expansion

$$
\hat{\boldsymbol{u}}(s)=R(s ; L) \hat{\boldsymbol{f}}(s)=\sum_{k=1}^{n} \mu_{k}(s) \boldsymbol{u}_{k}(s)\left(\boldsymbol{f}_{k}(s), \hat{\boldsymbol{f}}(s)\right), \quad s \in \rho(L),
$$

converges, whereas orthogonality $\left(f_{k}(s), f_{l}(s)\right)=\left(\boldsymbol{u}_{k}(s), \boldsymbol{u}_{l}(s)\right)=\delta_{k l}$ holds and $\mu_{1}(s) \geq$ $\mu_{2}(s) \geq \cdots \geq \mu_{n}(s)>0$ (Riesz \& Sz.-Nagy 1956, p. 203; Kato 1980, pp. 160-161 and 260-262). The pair $\left\{\boldsymbol{u}_{k}(s), \boldsymbol{f}_{k}(s)\right\}$ defines a hierarchy of rank-1 operators and $\mu_{k}(s)$ is referred to as singular value. From a physics point of view, each pair defines the radial pattern of the $k$ th-optimal response $\boldsymbol{u}_{k}(s)$ to forcing $\boldsymbol{f}_{k}(s)$. We call $\boldsymbol{f}_{k}(s), \boldsymbol{f}_{k}(s)$ forcing and response structures, respectively. The respective singular values $\mu_{k}(s)$ signify the $k$ th-optimal energy amplifications and the leading singular value is identical to the norm of the resolvent $\mu_{1}^{2}(s)=\|R(s ; L)\|^{2}$ which can be interpreted as the maximum amplification obtained for all admissible forcing fields (Riesz \& Sz.-Nagy 1956, p. 149).

The canonical decomposition (3.1) is inherently related to the respectively self-adjoint eigenvalue problems (Kato 1980, p. 261)

$$
R^{\dagger} R \boldsymbol{f}_{k}=\mu_{k}^{2} \boldsymbol{f}_{k} \quad \text { and } \quad R R^{\dagger} \boldsymbol{u}_{k}=\mu_{k}^{2} \boldsymbol{u}_{k} \quad \text { with } \boldsymbol{u}_{k}:=\mu_{k}^{-1} R \boldsymbol{f}_{k}, \mu_{k} \neq 0 .
$$

If $R(s ; L)$ is normal the two eigenvalue problems can be identified, implying that forcing and response are structurally identical. By definition 2.1, receptivity relies on perturbation internalisation, hence, forcing and response structures must have different spatial support. This is possible if the resolvent is non-normal; cf. conjecture 3.1. The degree of non-normality can be estimated from bounds on the resolvent norm.

Let $\phi(L):=\operatorname{cl}\left\{s \in \mathbb{C} \mid s=(\boldsymbol{q}, P L \boldsymbol{q}), \boldsymbol{q}=(\boldsymbol{u}, p)^{T}\right.$ such that $\left.\operatorname{div} \boldsymbol{u}=\operatorname{div} B^{\dagger} L \boldsymbol{q}=0,\|\boldsymbol{u}\|=1\right\}$ be the closure $\operatorname{cl}\{\cdot\}$ of the numerical range (Kato 1980, p. 267; Gustafson \& Rao 1997, p. 1). Then, for all $s \in \rho(L)$ which are in the complement of $\phi(L)$,

$$
\frac{1}{d(s, \sigma(L))} \leq\|R(s ; L)\| \leq \frac{1}{d(s, \phi(L))},
$$

where $d(s, \sigma(L)):=\inf _{\lambda \in \sigma(L)}|s-\lambda|>0$ defines the distance of $s \in \rho(L)$ from the closest element in the spectrum and analogously for $d(s, \phi(L))$ with $s \notin \phi(L)$ (Kato 1980, 
thereom 3.2; Gustafson \& Rao 1997, (4.6)-(7) and lemma 6.1-4). Equality with the lower bound holds in (3.3) if the resolvent is normal (Kato 1980, pp. 272-277).

From a physical point of view, the left-hand side of (3.3) describes the 'classical' resonance behaviour of the equivalent normal operator (solely determined by its spectrum) as the excitation frequency $s$ differs from elements of the spectrum (Arnol'd 1992, p. 235). In contrast, non-normal operators are principally amenable to significant amplification even far from the spectrum (Trefethen \& Embree 2005, p. 10). Contours of the resolvent norm (i.e. the pseudospectrum) therefore represent generalised resonance (pseudo-resonance) of the system. Comparison of the graphs of the lower bound with the resolvent norm along the imaginary axis $(s=\mathrm{i} \omega)$ therefore reveals frequency ranges where the resolvent is non-normal and thus pseudo-resonance outweighs 'classical' resonance (cf. figure 3).

The right-hand side of (3.3), defining the distance to the numerical range $\phi(L)$, is physically not associated with resonance but related to the capacity of energy growth which we use in $\S 4.1$ to derive the location of the instantaneously most amplified perturbation. Despite identical structure of the two bounds in (3.3), it should be emphasised that we cannot use the upper bound to draw a meaningful graph (similar to figure $3 a$ ) which bounds the pseudo-resonance $\|R(s=\mathrm{i} \omega ; L)\|$ from above along the imaginary axis. Rather, the intention is to gain insight into non-normality from patterns the linear operator $L$ defines in the complex plane. The smallest set characterising $L$ (sufficient for the dynamics of normal operators) is the spectrum $\sigma(L)$ while the numerical range $\phi(L)$ is the largest set determining dynamics. Pseudospectra $\sigma_{\epsilon}(L) \subset \mathbb{C}$, determining the transient dynamics, continuously fill the gap whereas $\lim _{\epsilon \rightarrow 0} \sigma_{\epsilon}(L) \leftrightarrow \sigma(L)$ and $\lim _{\epsilon \rightarrow \infty} \sigma_{\epsilon}(L) \leftrightarrow \phi(L)$ (Gustafson \& Rao 1997, p. 106; Trefethen \& Embree 2005, p. 172). We expect that resolvent non-normality can be inferred from differences in these sets.

\section{Selective non-normality of linear vortex dynamics}

Hill (1995, p. 183) noted that receptivity is determined by geometry, physical location and frequency of the source as well as the reference-flow characteristics. Indeed, wind-tunnel and numerical experiments provide evidence that vortices favour a response at distinguished frequencies and to particular disturbance patterns (e.g. Marshall \& Beninati 2005; Bailey et al. 2018). Adopting the linear model developed in $\S 2.3$ the last of Hill's points is obvious since the formal operator is essentially determined by the reference-flow profile as discussed below. Further assessment shows that the reference flow also imposes a preferred position and frequency for disturbances contributing to free-stream receptivity; we find that vortices are generally most susceptible to the archetypal forcing pattern of coiled filaments aligned with the critical layer. As stated in conjecture 3.1 non-normality is a necessary requirement for linear receptivity to free-stream turbulence and hence to quantify the above aspects; we thus conclude the main result of this section:

CONJECTURE 4.1. Linear perturbation dynamics about axisymmetric vortices satisfying $\boldsymbol{U}(r)=U_{\theta}(r) \boldsymbol{e}_{\theta}+U_{z}(r) \boldsymbol{e}_{z}$ is governed by an $\omega$-selectively non-normal linear operator. In the inviscid limit, non-normality is maximised for critical-layer perturbations.

\subsection{Analysis of the formal operator and its numerical range}

To the best of our knowledge no analytic expression of the resolvent for three-dimensional inhomogeneous perturbations about smooth viscous vortices exists today (e.g. Ash \& Khorrami 1995, p. 321; Roy \& Subramanian 2014, p. 439). However, it can be shown that 
normality of a linear operator implies normality of its resolvent (Kato 1980, pp. 276-277) so that we proceed by analysing $L$, expecting similar properties to hold for $R(\mathrm{i} \omega ; L)$, too.

In order to attribute non-normality a physical significance, the linear operator in (2.3) is written as the sum

$$
L=\nabla_{U}+\nabla U+A
$$

comprising contributions from advection $\nabla_{U}$, mean-velocity gradient $\nabla U$ and the Stokes operator $A$ (governing the Stokes system; Sohr 2001, § 4), respectively. Pressure gradient and continuity equation are contained in the Stokes operator by definition.

The formal adjoint of (4.1) is defined through the Lagrange identity (Friedman 1962, p. 148) and reads

$$
L^{\dagger}=\nabla_{U}^{\dagger}+(\nabla U)^{\dagger}+A^{\dagger}=-\nabla_{U}+(\nabla U)^{\dagger}+A
$$

Comparing (4.1) and (4.2) element-wise, it is evident that the Stokes operator $A$ is (formally) self-adjoint and hence normal. Taken independently, the advection operator $\nabla_{U}$ is formally skew-adjoint, thus also normal. (A skew-adjoint operator generates a unitary (inner-product preserving) propagator, viz. $T(t)^{\dagger}=T(t)^{-1}$ for all $t$ (Engel \& Nagel 2000, p. 20).) While the dynamics on unbounded or periodic fluid domains (in the direction of the mean flow) seems to promote actual self-adjointness, realisations on bounded domains are associated with (inflow-outflow) boundary conditions which break the formal behaviour. In terms of physics, this latter advective non-normality known in the global approach manifests as the spatial separation (in $z$ ) of the direct and adjoint eigenfunctions (Sipp et al. 2010, p. 7). From the three terms in (4.1) only the velocity-gradient operator $\nabla \boldsymbol{U}$ is inherently non-normal in isolation.

Since the Stokes operator is normal (independently) and increasing viscosity consistently found to dampen non-normal dynamics, non-normality of $L$ should result from the inviscid advection and mean-velocity-gradient operators (Antkowiak 2005, p. 3; Pradeep \& Hussain 2006, p. 279; Heaton \& Peake 2007, p. 278). It should be noted though that the sum of normal operators is not necessarily normal, as shown e.g. for the advection-diffusion operator by Reddy \& Trefethen (1994, p. 1647).

Neglecting $A$ in (4.1) and (4.2), formal non-normality of the advection-velocity-gradient operator is associated with the commutator $\left[L, L^{\dagger}\right] \rightarrow\left[\nabla_{U}, 2 S\right]+\left[\nabla U,(\nabla U)^{\dagger}\right]$ (recalling that advection is formally normal) where $S:=\left(\nabla U+(\nabla U)^{\dagger}\right) / 2$ denotes the Hermitian part of the velocity gradient. Explicitly,

$$
\left[L, L^{\dagger}\right] \rightarrow\left[\begin{array}{ccc}
-\left(W_{z}+2 \Omega\right) r \mathrm{~d} \Omega / \mathrm{d} r-\left(\mathrm{d} U_{z} / \mathrm{d} r\right)^{2} & 0 & 0 \\
0 & \left(W_{z}+2 \Omega\right) r \mathrm{~d} \Omega / \mathrm{d} r & \Omega \mathrm{d} U_{z} / \mathrm{d} r \\
0 & \Omega \mathrm{d} U_{z} / \mathrm{d} r & \left(\mathrm{~d} U_{z} / \mathrm{d} r\right)^{2}
\end{array}\right] .
$$

Writing $\left(W_{z}+2 \Omega\right) r \mathrm{~d} \Omega / \mathrm{d} r=W_{z}^{2}-(2 \Omega)^{2}$ readily shows that the dynamics is formally normal if the reference flow is that of a rigid-body rotation and translation (i.e. $W_{z}=$ $2 \Omega$ and $U_{z}=$ const.). In other words, non-normality is unaffected by the superposition of rotation or translation as a rigid body and, in particular, indistinguishable for observers being in rigid-body rotation or translation to one another, e.g. between aeroplane cruise condition and laboratory experiment.

To get a deeper understanding of the resolvent non-normality, let us now consider the upper bound in (3.3), which, as we re-emphasise, is not amenable to the same physical 


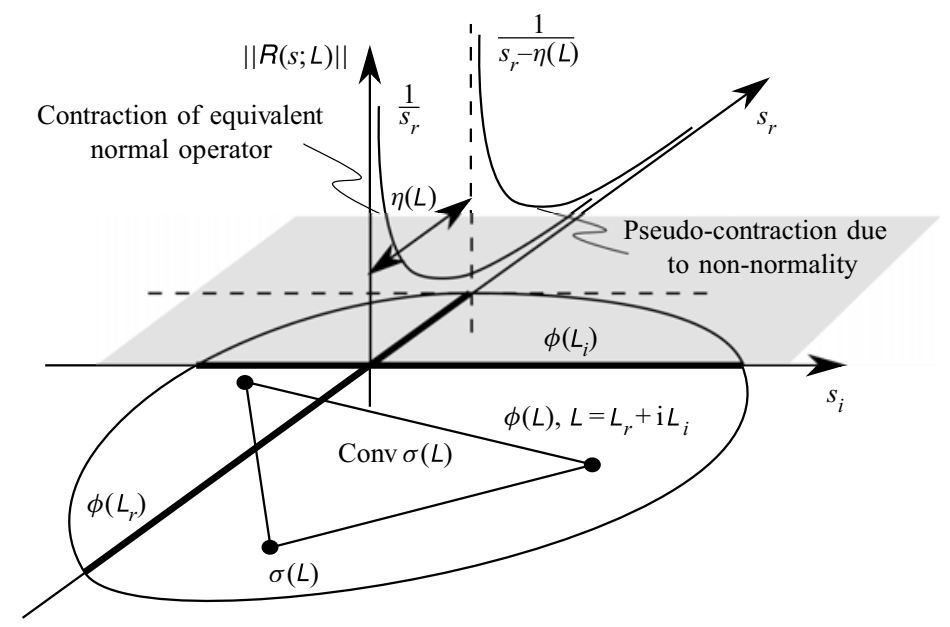

FIGURE 1. Schematic comparison of the dissipative dynamics (contraction) generated by the equivalent normal operator (i.e. solely defined by its spectrum $\sigma(L)$ and its convex hull conv $\sigma(L)$ ) with the actual dynamics (determined by the numerical range $\phi(L)$ ) showing that non-normality is associated with the numerical abscissa $\eta(L)$ (see (4.5)). The unstable half-plane is visualised by grey shading.

and graphical interpretation as the lower bound in terms of resonance but rather the intention is the assessment of non-normality. In fact, the upper bound merely means that the pseudospectrum $\sigma_{\epsilon}(L)$ cannot be much larger than the numerical range $\phi(L)$ (Trefethen $\&$ Embree 2005, p. 169). Figure 1 shows a qualitative sketch illustrating the principal terminology used thereafter.

We write $L=L_{r}+\mathrm{i} L_{i}$ with the Hermitian part $L_{r}:=\left(L+L^{\dagger}\right) / 2$ and the skew-Hermitian part $L_{i}:=\left(L-L^{\dagger}\right) /(2 \mathrm{i})$, respectively. This decomposition implies the inclusion the spectrum in the numerical range, $\sigma(L) \subset \phi\left(L_{r}+\mathrm{i} L_{i}\right) \subset \phi\left(L_{r}\right)+\mathrm{i} \phi\left(L_{i}\right)$, which is shown in figure 1 (Kato 1980, pp. 309-310; Gustafson \& Rao 1997, pp. 6, 103). From a physical point of view, the Hermitian part $L_{r}=S+A$ determines energy growth while the skew-Hermitian part $L_{i}=-\mathrm{i} \nabla_{U}+W(\nabla U=S+\mathrm{i} W$ where $W$ is the skew-Hermitian mean-vorticity operator) is associated with conservative redistribution (cf. also the remark in the context of $\nabla_{U}$ above). In agreement, Pradeep \& Hussain (2006, p. 264) conclude that mean vorticity promotes vortex waves which do not contribute to energy growth. A priori this does not tell us anything about the origin of non-normality, however, our interest in energy amplification suggests closer examination of $\phi\left(L_{r}\right)$.

This reasoning is reflected in the Hille-Yosida generation theorem (Engel \& Nagel 2000, pp. 73-76). Let $c \in \mathbb{R}$ be a constant (which we identify with the numerical abscissa $\eta(L)$ below), then the propagator $T(t)$ is a pseudo-contraction if and only if

$$
\|T(t)\| \leq \mathrm{e}^{c t} \quad \forall t \geq 0 \Leftrightarrow\|R(s ; L)\| \leq \frac{1}{s_{r}-c}, \quad s_{r}>c .
$$

By definition, the associated generator $L$ is contractive if its numerical range does not protrude into the unstable half-plane while it is pseudo-contractive if it becomes contractive upon a constant shift $L-c$ (Kato 1980, pp. 278-279; Engel \& Nagel 2000, p. 75). Physically, a contractive propagator represents dissipation (of energy; $c=0 \Rightarrow$ $\|T(t)\| \leq 1)$ whereas a pseudo-contraction is dissipative beyond a certain threshold $c$. 
If $L$ was a normal operator, the numerical range would be the convex hull of its spectrum $\phi(L)=\operatorname{conv} \sigma(L)$ as sketched in figure 1 (Gustafson \& Rao 1997, theorem 1.4-4). Since the considered vortices are asymptotically stable this would imply $c=0$ in (4.4) and the equivalent normal operator would describe pure dissipation. The present deviation from this equivalent normal dynamics is a consequence of non-normality and in a sense proportional to the protrusion of the numerical range into the unstable half-plane. The maximum protrusion (recall that $\operatorname{div} \boldsymbol{u}=0$ )

$$
\eta(L):=\sup _{s \in \phi(L)} s_{r}=\sup _{\boldsymbol{u} \neq 0} \frac{\left(\boldsymbol{u}, L_{r} \boldsymbol{u}\right)}{\|\boldsymbol{u}\|^{2}}=\sup _{\boldsymbol{u} \neq 0} \frac{(\boldsymbol{u},(S-v \Delta) \boldsymbol{u})}{\|\boldsymbol{u}\|^{2}}
$$

is called the numerical abscissa (Trefethen \& Embree 2005, p. 174). The operator $S-v \Delta$ in (4.5) is self-adjoint and the numerical abscissa is identical to its largest eigenvalue. Since the viscous term is necessarily negative (Sohr 2001, p. 101) and by the above remarks on the damping effect of viscosity for the non-normal dynamics, we assume an inviscid fluid in the following.

In terms of physics, the numerical abscissa represents the maximum instantaneous energy growth. Let $v=0$ in (4.5), then the momentary change of integral energy is governed by the inviscid Reynolds-Orr equation for all $t \geq 0$ (Joseph 1976, p. 10)

$$
\frac{\mathrm{d}}{\mathrm{d} t} \frac{\|\boldsymbol{u}(t)\|^{2}}{2}=\left(\boldsymbol{u}(t), \frac{1}{2}\left[\begin{array}{ccc}
0 & r \mathrm{~d} \Omega / \mathrm{d} r & \mathrm{~d} U_{z} / \mathrm{d} r \\
r \mathrm{~d} \Omega / \mathrm{d} r & 0 & 0 \\
\mathrm{~d} U_{z} / \mathrm{d} r & 0 & 0
\end{array}\right] \boldsymbol{u}(t)\right), \quad\|\boldsymbol{u}(0)\|=1
$$

assuming the generic reference flow $U(r)=U_{\theta}(r) \boldsymbol{e}_{\theta}+U_{z}(r) \boldsymbol{e}_{z}$. Searching for the maximum of (4.6), the right-hand side is seen to coincide with the definition of the numerical abscissa (4.5) in the limit as $t \rightarrow 0$. This is equivalent to $\mathrm{d}\|T(t \rightarrow 0)\| / \mathrm{d} t=$ $\eta(L)$ where the numerical abscissa is the largest eigenvalue of $S$ for an inviscid fluid (see also Trefethen \& Embree 2005, theorem 17.4). Comparing (4.6) with the commutator (4.3) confirms that energy growth in an asymptotically stable system is possible only for reference states which are not in rigid-body motion (cf. also Joseph 1976, p. 10). The actual maximum energy-amplification capacity serves as a measure to assess non-normality.

The Hermitian part of the velocity gradient is self-adjoint, hence, the spectral theorem guarantees existence of real eigenvalues $\lambda_{1}=0, \lambda_{2,3}:= \pm \lambda= \pm \sqrt{(r \mathrm{~d} \Omega / \mathrm{d} r)^{2}+\left(\mathrm{d} U_{z} / \mathrm{d} r\right)^{2}} / 2$ and mutually orthogonal eigenvectors

$$
\boldsymbol{v}_{1}=\left(\begin{array}{c}
0 \\
-\mathrm{d} U_{z} / \mathrm{d} r \\
r \mathrm{~d} \Omega / \mathrm{d} r
\end{array}\right), \quad \boldsymbol{v}_{2,3}=\left(\begin{array}{c} 
\pm 2 \lambda \\
r \mathrm{~d} \Omega / \mathrm{d} r \\
\mathrm{~d} U_{z} / \mathrm{d} r
\end{array}\right)
$$

Physically, the eigenvectors $(4.7 a, b)$ span three orthogonal eigenspaces for which the production bilinear form in (4.6) vanishes (zero strain) and is negative/positive, respectively. The maximum eigenvalue corresponds to the numerical abscissa which is attained if the perturbation projects identically on the associated eigenvector $\boldsymbol{v}_{3}$.

Eigenvectors $\boldsymbol{v}_{2,3}$ only differ in the sign of the radial component while their projections onto a cylinder of radius $r, \boldsymbol{v}:=\boldsymbol{v}_{2,3}-\boldsymbol{e}_{r}\left(\boldsymbol{e}_{r}, \boldsymbol{v}_{2,3}\right)$ say, are identical and are conveniently represented in terms of their streamlines $r \mathrm{~d} \theta / v_{\theta}=\mathrm{d} z / v_{z}$. On the other hand, an analogous representation on the cylinder holds for arbitrary perturbations $\hat{\boldsymbol{u}}$ and the streamlines 
take on the form of helices due to the assumed symmetry. The pitch of the perturbation streamlines (streamwise increment $\mathrm{d} z$ per azimuthal increment $r \mathrm{~d} \theta$ ) defines the angle

$$
\frac{1}{r} \frac{\mathrm{d} z}{\mathrm{~d} \theta}=-\frac{m}{r \alpha} \quad \text { while } \quad \frac{1}{r} \frac{\mathrm{d} z}{\mathrm{~d} \theta}=\frac{1}{r} \frac{\mathrm{d} U_{z} / \mathrm{d} r}{\mathrm{~d} r \Omega / \mathrm{d} r}
$$

is the streamline angle of the eigenvector projection. In order that perturbation and eigenvector align, it is necessary that the above angles match, i.e.

$$
-\frac{m}{r \alpha}=\frac{1}{r} \frac{\mathrm{d} U_{z} / \mathrm{d} r}{\mathrm{~d} r \Omega / \mathrm{d} r} \Leftrightarrow \frac{\mathrm{d}}{\mathrm{d} r}\left(m \Omega+\alpha U_{z}\right)=0 \Leftrightarrow m \Omega+\alpha U_{z}=\omega=\text { const. }
$$

which is precisely the critical-layer condition (cf. $\$ 2.3$ ). Perturbation alignment on the cylinder is necessary but not sufficient for energy growth. It is in fact the radial component $\pm \lambda$ in $(4.7 a, b)$ that decides whether energy is amplified or attenuated. The situation of energy attenuation through critical-layer perturbations is known as Landau damping (Antkowiak 2005, p. 13; Fabre et al. 2006, p. 255).

The importance of stationary values of $m \Omega(r)+\alpha U_{z}(r)-\omega$ for inviscid instability was previously shown by Leibovich \& Stewartson (1983) (see also Ash \& Khorrami 1995 , p. 332). Approximate alignment of viscous and inviscid instability modes with the principal eigenvector was shown by Abid (2008, p. 28) for the Batchelor vortex and increases with $q \leq 1$ (see $(2.1 a-c)$ ). Nevertheless, while perturbation alignment has been identified as the condition for maximum energy growth before, it seems that equivalence with the critical-layer condition (4.9) has not been stated explicitly, yet. Moreover, we are not aware of any previous result relating critical-layer perturbations directly to non-normality.

In order to quantify non-normality for Batchelor (Lamb-Oseen) and Moore-Saffman vortices, figure 2 shows graphs of the mean profiles $U_{z}(r), \mathrm{d} U_{z}(r) / \mathrm{d} r, \Omega(r)$ and $W_{z}(r)$.

With regards to the axial mean velocity and its gradient, shown in figure $2(a)$ for the Batchelor and Moore-Saffman vortex, the most essential aspect for the present work is the substantial localisation in the vortex core. For the Batchelor vortex $(2.1 a-c), U_{z}^{B}$ is exponentially confined to the core. For the Moore-Saffman vortex $U_{z}^{M S}(r) \sim\left(n^{-1}-1\right)$ $r^{-2 n}$ as $r \rightarrow \infty$ holds by definition (Moore \& Saffman 1973, (3.5)). Nevertheless, jet-wake coexistence renders this asymptotic irrelevant for the practically pertinent behaviour in the core vicinity where the Moore-Saffman vortex behaves effectively identically to the Batchelor vortex. This substantial confinement of the axial mean velocity is in agreement with its importance for the discrete spectrum (discussed in § 4.2) and suggests negligible pertinence for disturbances located in the free stream. The critical-layer location for peak amplification of the Batchelor (Lamb-Oseen) vortex $(m=1, \omega=0.1, q=4)$ is indicated by a straight line at $r_{c} \approx \pi$ where no measurable effect of the axial mean flow is to be expected any more.

The second direct source of non-normality is by differential mean angular velocity $\Omega \neq$ const. and mean streamwise vorticity $W_{z}$, shown in figure $2(b)$. Vorticity is again substantially localised in the core, obeying an exponential law for the Batchelor (Lamb-Oseen) vortex $(2.1 a-c)$ and qualitatively similar behaviour for the Moore-Saffman vortex. From the characteristic reference-flow profiles shown in figure 2, angular velocity $\Omega$ is the only quantity which is not (almost) exponentially decreasing. As a matter of fact, $\Omega(r) \sim r^{-n-1}$ as $r \rightarrow \infty$ holds for all models, whereas $n=1$ corresponds to the Batchelor (Lamb-Oseen) vortex (Moore \& Saffman 1973, (3.5)) and $n=0.75$ is a lower bound for the Moore-Saffman vortex fitting experimental trailing vortices (see $§ 2.2$ ). 
(a)

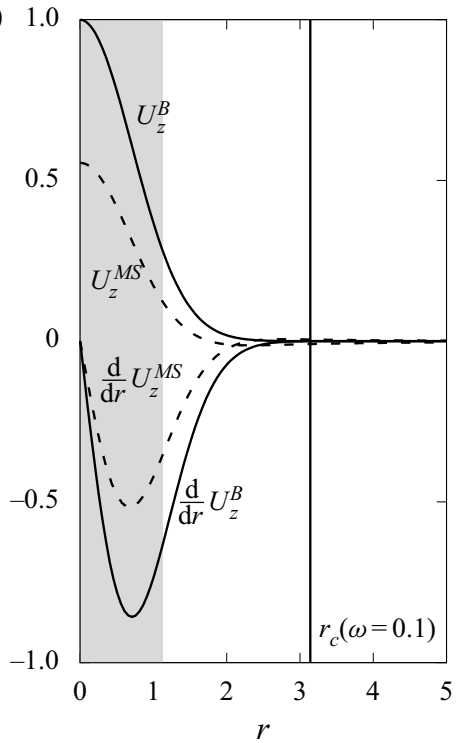

(b)

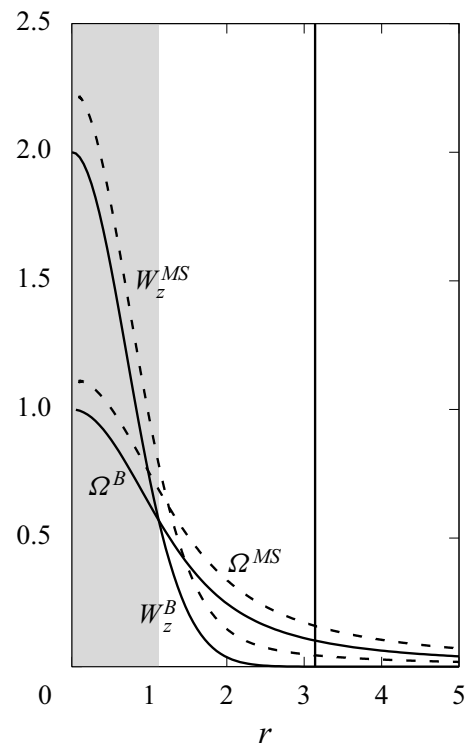

FIGURE 2. Comparison of radial mean-flow profiles for Batchelor (B) and Moore-Saffman (MS) vortices $(n=0.75)$. Axial velocity and mean-shear profiles in $(a)$ show (at least visually) exponential localisation in the core region, restricting significant contribution to $r \lesssim 2$. Mean angular velocity $\Omega$ and axial vorticity $W_{z}$ in $(b)$ show essentially identical behaviour for the two models, the latter being also strongly localised. For reference the vortex core is shown in grey shading as well as the critical layer $r_{c}$ of the Lamb-Oseen vortex for $\omega=0.1, m=1$.

We conclude that receptivity to the free stream should be largely independent of the vortex model and subsequent discussion will focus on the Lamb-Oseen vortex. Variation of the reference flow will be further discussed in $\S 5$.

\subsection{Selective non-normality of the Lamb-Oseen vortex}

According to the left-hand side of (3.3), the difference between the resolvent norm and the reciprocal of the shortest distance of any given frequency $\mathrm{i} \omega \in \rho(L)$ to the spectrum is a local measure for non-normality. Comparing graphs of these two functions we find vortices to be effectively non-normal only on a narrow frequency band (termed $(\omega$-)selective non-normality).

All results of this section are obtained for the Lamb-Oseen vortex and $R_{\Gamma}=5000$, $m=1, \alpha=1.55$ as in Guo \& Sun (2011, p. 3191). By (3.1) a compact resolvent can be expanded in a convergent series of rank-1 operators, weighted by the associated singular value. We find the singular values to be rapidly decreasing for all considered frequencies, thus, restricting to leading-order structures henceforth (constituting the rank-1 approximation of the resolvent by (3.1)). It should be noted though that this is not true for steady perturbations $(\omega \approx 0)$ due to the continuous spectrum $\sigma_{c}^{\infty}(L)$ introduced below.

Figure $3(b)$ shows nested isocontours of the pseudospectrum in the complex $s$-plane for values of $\epsilon=\|R(s ; L)\|^{-1} \in\left\{10^{n}: n=-1,-1.5,-2,-2.5,-3,-4,-5\right\}$, effectively approaching the spectrum shown by dots (for details on pseudospectra see e.g. Trefethen \& Embree 2005). The particular case of $s=\mathrm{i} \omega$ is shown in figure 3(a) and will be discussed thereafter. 

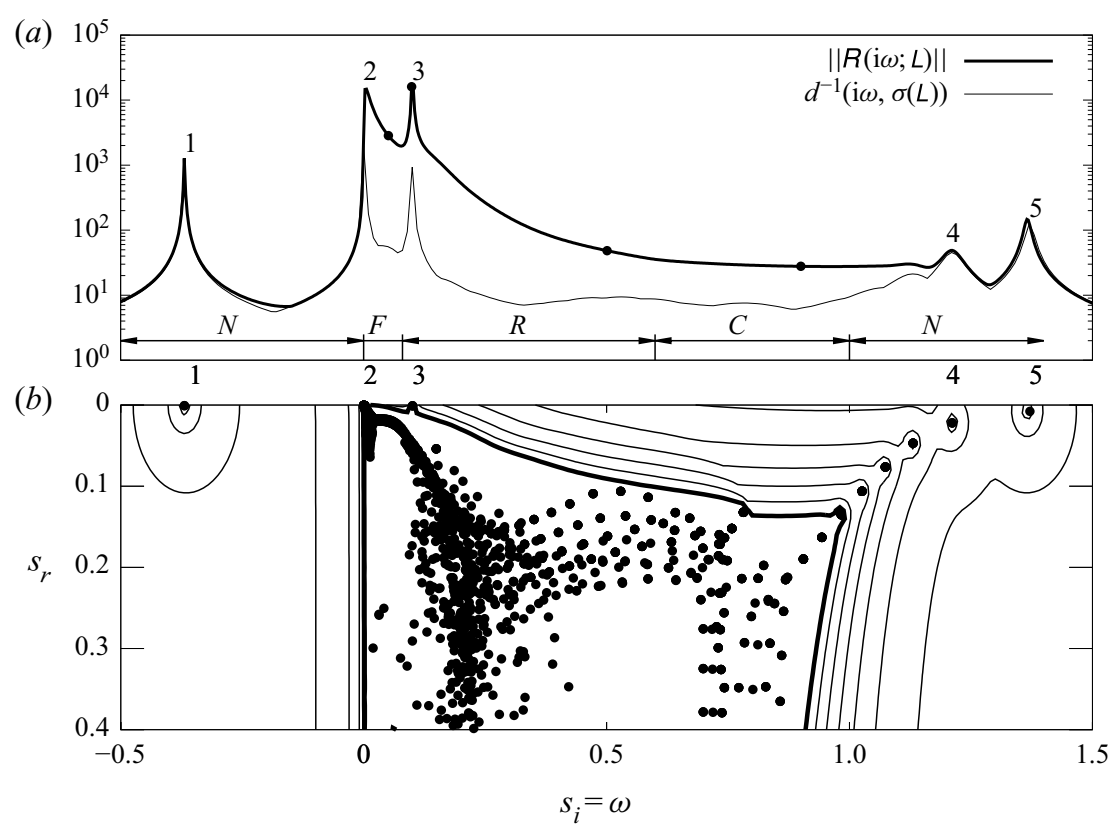

FIGURE 3. Comparison of the resolvent norm for the Lamb-Oseen vortex ( $m=1, \alpha=1.55$, $\left.R_{\Gamma}=5000\right)$ with the reciprocal distance in $(a)$ assuming a harmonic ansatz $\omega=s_{i} \in \mathbb{R}$ and $s_{r}=0$, revealing $\omega$-selective non-normality approximately for $0 \lesssim \omega \lesssim 1$. Distinction of dynamical regimes of the resolvent: $N$ signifying a nominally normal operator and $\{F, R, C\}$ being associated with non-normality due to critical-layer singularity. The latter distinguishes the dynamics essentially situated in the far field $(F)$ and core $(C)$, respectively, while only the intermediate range critical-layer forcing $(R)$ is associated with receptivity. Solid dots mark frequencies of forcing/response structures shown in figure 4. Numbering refers to the (pseudo)spectrum in $(b)$, showing nested isocontours of the resolvent norm and the spectrum (solid dots) in the complex $s$-plane.

It should be noted that the spectrum is organised into the same branching structure as the spectrum shown in Fabre et al. (2006, figure 7) for the Lamb-Oseen vortex with $m=1$, $\alpha=3$ and $R_{\Gamma}=1000$. Even more, qualitatively the same pseudospectrum and eigenvalue scattering is reported in Mao \& Sherwin $(2011$, p. 8) for the Batchelor vortex with $m=0$, $\alpha=10, R_{\Gamma} \approx 2000$ and $q=3$. These findings provide further support for conjecture 5.1 that the linear vortex dynamics is generic as discussed in $\S 5$.

The spectrum of the linear operator $L$ governing the three-dimensional perturbation dynamics about vortices comprises contributions from discrete eigenvalues $\sigma_{d}(L)$ as well as a (semi-infinite) continuous spectrum $\sigma_{c}^{\infty}(L)$ due to spatial unboundedness. The latter is argued to be $\sigma_{c}^{\infty}(L)=\left\{s=s_{r}+\mathrm{i} s_{i} \in \mathbb{C} \mid \alpha^{2} v<s_{r}<\infty, s_{i}=0\right\}$ (Fabre et al. 2006, appendix A; Mao \& Sherwin 2011, p. 14 and appendix B) and can be anticipated from eigenvalue and contour clustering along the real axis in figure $3(b)$. Receptivity of the Batchelor vortex to axisymmetric disturbances has previously been related to long-wavelength generalised eigenmodes pertaining to $\sigma_{c}^{\infty}(L)$ which penetrate into the core (Mao \& Sherwin 2011, pp. 1-10 and figure 3). We exclude this mechanism by restricting to finite kinetic energy solutions and rather emphasise remote receptivity without mass transport across the system boundary (cf. discussion at the end of $\S 2.1$ ). Rather $\sigma_{c}^{\infty}(L)$ is an artefact of the mathematical model of an unbounded domain and the 
associated perturbations are considered irrelevant here (cf. also Heaton \& Peake 2007, pp. 275-295).

Considering an inviscid fluid, an additional inviscid continuous spectrum $\sigma_{c}^{0}=\{s \in$ $\left.\mathbb{C} \mid s_{i}=\omega=m \Omega(r), s_{r}=0\right\}$ exists as a consequence of a critical-layer singularity of the homogeneous problem (Le Dizès 2004, p. 319; Roy \& Subramanian 2014, § 3.2). For non-vanishing viscosity it degenerates to a discrete spectrum of a large number of stable discrete modes (Heaton \& Peake 2007, p. 282) which are algebraically localised in the core vicinity, referred to as potential modes by Mao \& Sherwin (2011, p. 2). This viscous remnant of the inviscid continuous spectrum, denoted $\sigma_{c}^{\nu}(L)$, is observed as the apparently random eigenvalue scattering in the rectangular central part of figure $3(b)$. Small values and shape of the $\left(\epsilon=10^{-5}\right)$-pseudospectrum (innermost thick contour in figure $3 b$ ) led Mao \& Sherwin (2011, p. 10) to speculate that the spectrum in fact remains continuous.

The distinguished situation of neutral harmonic perturbations corresponds to a cut at $s_{i}=\omega, s_{r}=0$ which yields the resolvent norm shown in figure $3(a)$ in comparison with the graph of the reciprocal distance. By (3.3) we observe the resolvent to be selectively non-normal in a frequency band of roughly $0 \lesssim \omega \lesssim 1$ while it is effectively normal outside this range. Considering the associated perturbation structures we will show in $\S 4.3$ that the non-normality frequency interval is essentially correlated with the critical layer, as already anticipated from analysis of the operator structure in $\S 4.1$.

Applying the same numbering in figure $3(a, b)$ indicates that peaks in the resolvent norm match with the least damped elements of the spectrum. Furthermore, the response modes associated with peaks 1, 4 and 5 belong to the D-, V-and C-families in the classification of Fabre et al. (2006) and are equivalent to the modes obtained from the eigenvalue problem for $L$. The associated peak forcing structures are identical to the eigenmodes of the adjoint $L^{\dagger}$. A thorough classification of perturbations in the $(\omega, \alpha)$-plane is postponed to $\S 5$ (cf. also figures 7-9).

These observations suggest that perturbations for which roughly $\omega \notin[0,1]$ the dynamics is governed by an effectively normal operator and hence irrelevant for receptivity according to definition 2.1 by conjecture 3.1. From a physical standpoint, the dynamic regimes labelled $N$ in figure 3(a) constitute classical resonance between congruent perturbation patterns, e.g. $\boldsymbol{f}_{1}\left(\omega_{l}\right) \cong \boldsymbol{u}_{1}\left(\omega_{l}\right)$ where $l \in\{1,4,5\}$ labels the peaks. Amplification away from the singularity is simply $d^{-1}\left(\mathrm{i} \omega,\left\{\omega_{l}\right\}\right), \omega \in \mathbb{R}$, in these cases (cf. (3.3)).

In order to gain further insight into the mechanisms of free-stream receptivity, let us now turn to the perturbation structures. Typical patterns of forcing-response pairs $\left\{\boldsymbol{u}_{1}(\omega), \boldsymbol{f}_{1}(\omega)\right\}$ for gradually increasing frequencies $\omega \in\{0.05,0.1,0.5,0.9\}$ are shown in the top and bottom row of figure 4 . Contours and colouring represent the streamwise component of the curl of the forcing and response structures. Magnitudes are understood to be qualitative and not uniform across panels to emphasise the relative locations and perturbation patterns. For this purpose a solid circle of radius $r_{1}=1.12$ indicates the vortex boundary, revealing that forcing structures are gradually located closer to the core as the frequency is increased. The forcing structure crosses the vortex boundary at a frequency of $\omega \approx 0.5$ and hence disqualifies perturbations from $\omega \gtrsim 0.6$ (say) from contributing to free-stream receptivity as defined in definition 2.1 for the absence of radial transport, despite local non-normality. Associated forcing-response pairs are not congruent but systematically located in the vortex core and, for this reason, are referred to as $C$-regime, cf. figure 3(a).

Significant amplification is observed for quasi-steady excitation $\omega \approx 0$ in figure $3(a)$. Considering the sequence $\omega \searrow 0$, the associated forcing and response structures are 
$\omega=0.05$

(a)

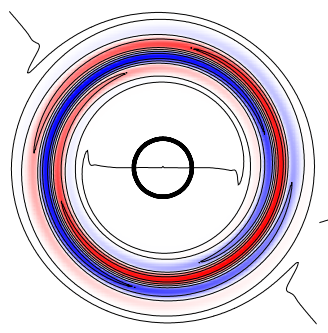

(b)

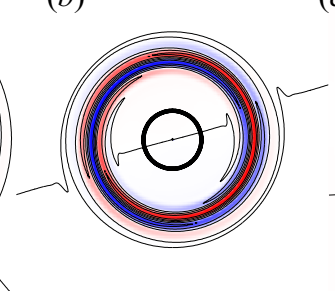

(c)

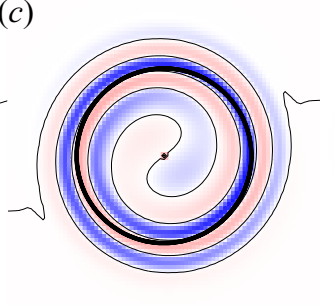

(d)

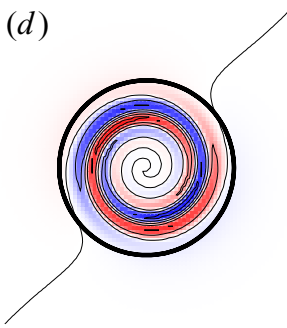

(e)

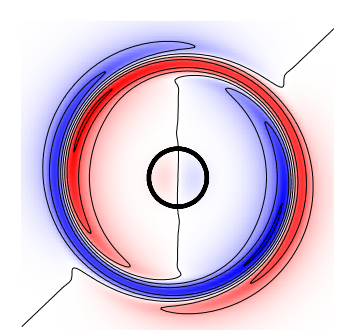

$(f)$

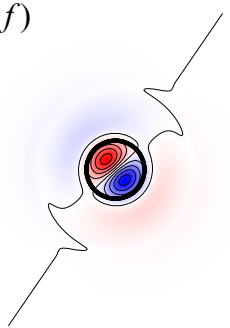

$(g)$

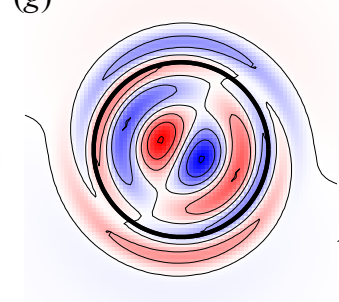

(h)

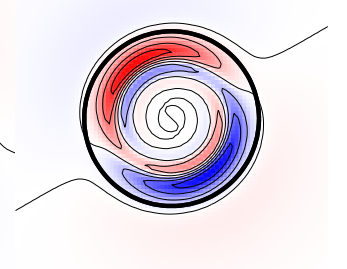

FIGURE 4. Streamwise component of $\operatorname{rot} f_{1}(\omega)$ and $\operatorname{rot} \boldsymbol{u}_{1}(\omega)$ for $\omega \in\{0.05,0.1,0.5,0.9\}$ (solid dots in figure $3 a)$ in $(a-d)$ and $(e-h)$, respectively, for the Lamb-Oseen vortex $(m=1$, $\left.\alpha=1.55, R_{\Gamma}=5000\right)$. Positive and negative values correspond to blue and red colouring, the scale not being chosen uniformly across different panels. The solid circle of radius $r_{1}=1.12$ indicates the vortex-core boundary.

found to be located at increasingly large radii as shown in figure 4 . The most important implication for receptivity is that below a certain frequency forcing and response structures take on the form of localised wave packets of comparable shape that are both far outside the vortex core, labelled $F$-regime in figure 3(a). This kind of forcing, although causing large amplification, is unable to cause (at least directly) core perturbations. For this reason it is assumed to be irrelevant for linear receptivity. The two cases of $F$ - and $C$-regimes underline that by conjecture 3.1 non-normality is necessary but not sufficient, the actual range of free-stream receptivity ( $R$-regime) is indicated in figure 3 . This considerable restriction of the receptivity frequency band suggests that vortices behave like strongly selective filters to free-stream turbulence (see also Antkowiak 2005, p. 72).

\subsection{Critical-layer alignment of the forcing structures}

As shown in $\S \S 4.1$ and 4.2 , the range of effective non-normality is essentially correlated with the inviscid continuous spectrum. The inviscid nature of the linear non-normal dynamics and the retained importance of the singular continuous spectrum even for a viscous fluid (cf. also Heaton \& Peake 2007, pp. 278-279, 287) is underscored by tracking the radial location of the forcing structures as a function of frequency, closely following the critical layer, in figure 5.

In $\S 2.3$ we recalled the definition of the critical layer as the radial location where mean advection equals perturbation propagation. Using the spatio-temporal Fourier ansatz of $\S 2.3$ the material-derivative operator (including streamwise transport for now) becomes a 
(a)

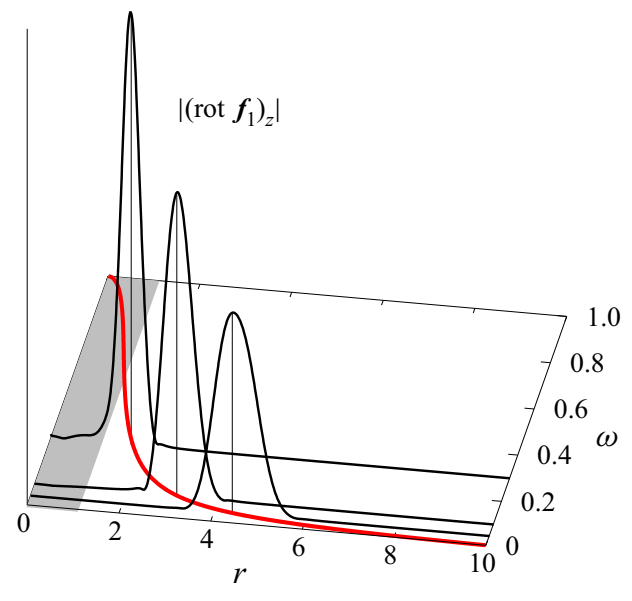

(b)

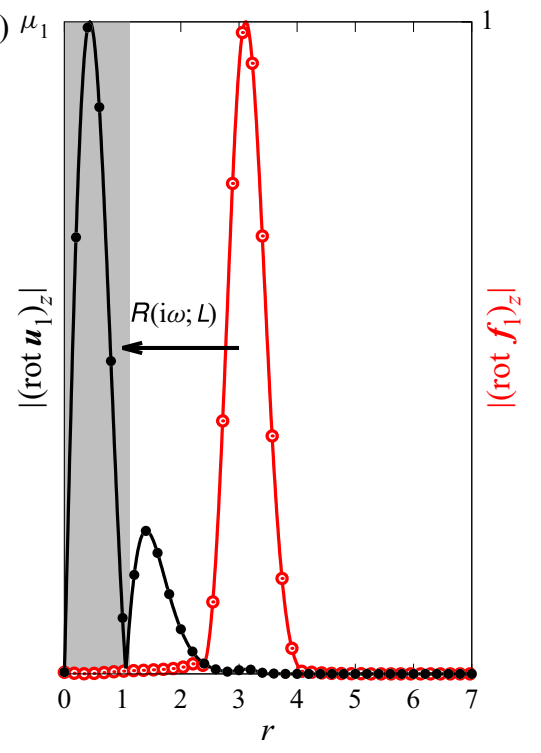

FIGURE 5. Archetypal critical-layer perturbations of the Lamb-Oseen vortex for $\alpha=1.55$, $m=1, R_{\Gamma}=5000$. Candidate wave frequencies $\omega$ in the critical layer (red line) and modulus of the streamwise component of rot $f_{1}(\omega)$ for selected forcing structures (thick black graphs) being centred around the critical layer and getting sharper as $\omega$ increases (as they approach the core) in $(a)$. Prototype of the critical-layer forcing-response pair at $\omega=0.1$ showing the perturbation shift from the critical layer into the vortex core in $(b)$. The shaded region signifying the vortex core delimited by $r_{1}=1.12$ in panels $(a, b)$.

multiplication operator associated with the symbol

$$
\frac{\partial}{\partial t}+\Omega(r) \frac{\partial}{\partial \theta}+U_{z}(r) \frac{\partial}{\partial z} \rightarrow m \Omega(r)+\alpha U_{z}(r)-\omega .
$$

Discussion in $\S 2.2$ suggests that $U_{z}$ is (nearly) exponentially localised in the core such that for disturbances in the free stream

$$
m \Omega(r)+\alpha U_{z}(r)-\omega \approx m \Omega(r)-\omega
$$

and receptivity to critical-layer forcing should be essentially independent from the axial mean velocity in practice. Thus, Lamb-Oseen, Batchelor and Moore-Saffman vortices are expected to have essentially identical critical-layer dynamics with regards to receptivity. Let $m=1$, the critical layer of the Lamb-Oseen vortex becomes the locus $\{r \in$ $(0, \infty) \mid \Omega(r)-\omega=0\}$, shown in figure $5(a)$ as a thick red line. The associated frequency range for critical-layer forcing is somewhat smaller than the range of non-normality shown in figure 3(a). Deviations from the Lamb-Oseen critical layer in figure $5(a)$ due to axial mean velocity (Batchelor or Moore-Saffman vortex) are essentially restricted to the core, unless $\alpha \gg 0$ or $q \rightarrow 0$. Both situations are excluded here since the trailing-vortex dynamics is of long wavelength (meandering) and dominated by mean rotation rather than axial velocity (i.e. jet behaviour).

Forcing structures in figure 5(a) are indeed systematically localised about the critical layer which strongly suggests a relation to the inviscid continuous spectrum and critical-layer forcing as the essential mechanism for receptivity. Archetypal free-stream 

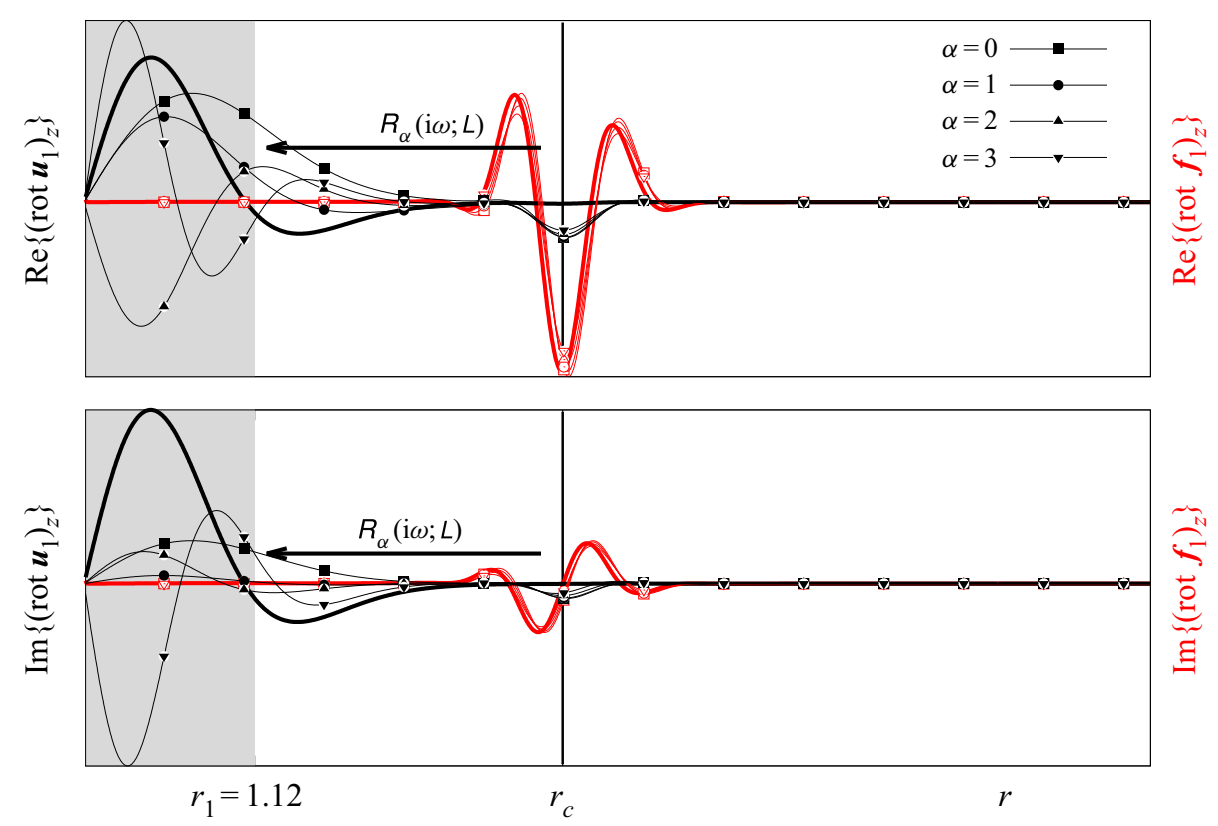

FIGURE 6. Real and imaginary part of the leading-order forcing and response structures (hollow red and solid black symbols, respectively) for the Lamb-Oseen vortex ( $\left.m=1, R_{\Gamma}=5000\right)$ at $\omega=0.1$ and $\alpha \in\{0,1,2,3\}$ in comparison with the archetype at $\alpha=1.55$ (thick line; cf. also figure 5). The characteristic forcing structures have essentially identical radial patterns in the vicinity of the critical layer at $r_{c} \approx \pi$. Resonantly excited forcing structures show comparable behaviour with spatial complexity increasing with $\alpha$.

receptivity is associated with the characteristic forcing-response patterns displayed in figure $5(b)$, showing the modulus of the streamwise curl component in figure 4 for $\omega=0.1$. The most important aspect qualifying this forcing-response pair as a candidate for free-stream receptivity is the fact that forcing and response structures have (almost) disjoint radial support, showing that vortices are susceptible to disturbances which do not physically penetrate the core.

The receptivity prototype shown in figures 4 for $\omega=0.1$ and $5(b)$, i.e. coiled forcing filaments in the critical layer resonantly exciting a core bending wave, is analogous to findings in transient-growth studies (Antkowiak \& Brancher 2004; Pradeep \& Hussain 2006). Its importance for receptivity to sustained (stochastic) forcing has been pointed out previously by Fontane et al. (2008, p. 250) who speculated that it might constitute a potential mechanism for vortex meandering (see also Viola et al. 2016, p. 545). While these two approaches formally discuss solutions to (2.3) in the time domain, the resolvent provides an analysis in frequency space (cf. (1.1)-(1.2) in $\S 1)$. All three approaches are mathematically related since the resolvent is the Laplace transform of the propagator (cf. § 1). All linear studies are complementary and analysis in frequency space (as opposed to time domain) has the same principal advantages and inconveniences as in the analysis of a time signal, for example. Specifically, it identifies those frequencies which contribute most to complex temporal dynamics (such as vortex meandering). As such, it is the natural framework for receptivity, characterising the vortex as a selective filter with frequency-dependent susceptibility. 

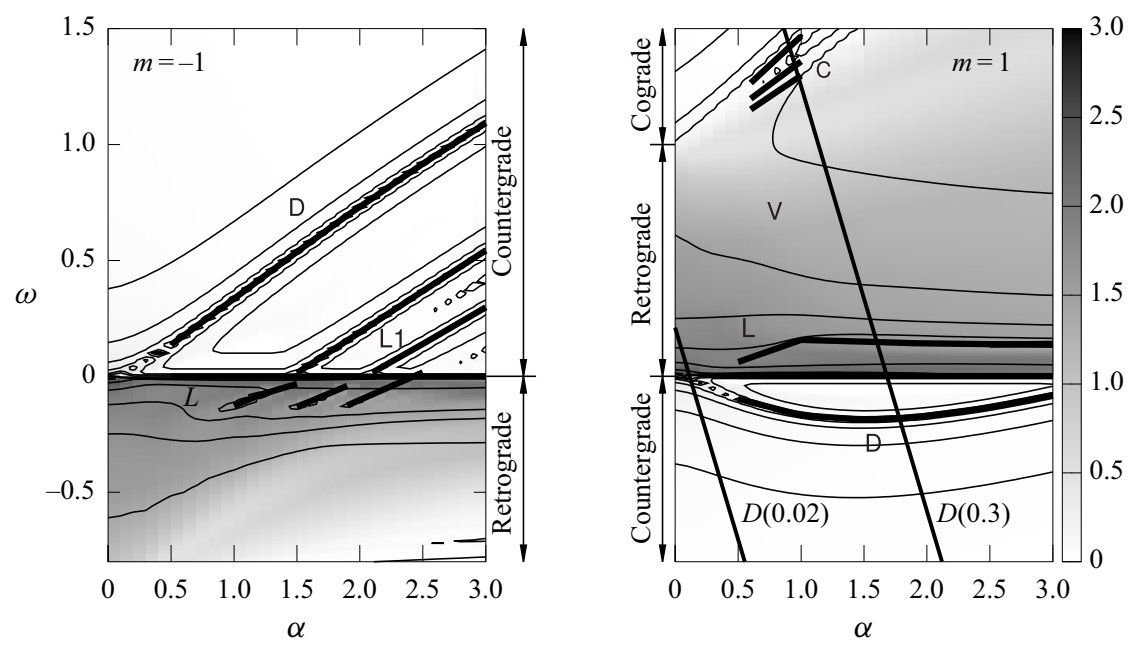

FIGURE 7. Resolvent norm of the Batchelor vortex in the $(\alpha, \omega)$-plane for $R_{\Gamma}=6000$ and $q=4$ showing (thin) nested contours for amplification levels $\left\{10^{n}: n=0.5,1.0,1.5, \ldots, 4.5\right\}$ peaking at the thick lines, comparing $m=-1(a)$ and $m=1(b)$. Superposition of the least damped eigenvalues obtained from solution of the eigenvalue problem (thick lines) reveals alignment with the peaks of the resolvent norm. Grey shading measures effective local non-normality defined by $\log _{10}\left\|R_{m, \alpha}(\mathrm{i} \omega ; L)\right\| / d\left(\omega, \sigma_{m, \alpha}(L)\right)^{-1} \in[0, \infty), \alpha \in[0,3]$, the lower bound being perfect local normality (white). Non-normality happens to be essentially correlated with the possibility of having critical-layer forcing. Inclined graphs $D=D\left(S_{m}\right)$ indicate the loci of candidate $(\omega, \alpha)$-pairs for the experimental meandering frequency $S_{m}$ (from Bailey et al. 2018, figure $7 a$ ) obtained from the Doppler relation.

\subsection{Variation of the axial wavenumber}

So far, (arbitrarily) fixing $\alpha=1.55$ we showed that vortices are receptive in a narrow frequency band to a particular disturbance pattern localised in a certain radial range of the free stream. Despite this selectivity in $\omega$ and $r$, we will provide evidence that receptivity is largely insensitive to variations in the axial wavelength. To illustrate that the choice of $\alpha$ is not essential for values on the considered interval, figure 6 shows the variation of forcing-response pairs taken for $\alpha \in\{0,1,2,3\}$ keeping $\omega=0.1=$ const. in comparison with the receptivity prototype at $\alpha=1.55$ (thick line). Apparently, forcing structures (red lines, hollow symbols) are almost indistinguishable for all cases and localised about the critical layer. The excited responses (black lines, solid symbols of same shape) all have radially disjoint support and are localised in the core (indicated by grey shading). Nevertheless, the complexity of the response, measured in terms of its roots, say, is seen to increase with the axial wavenumber.

This finding provides evidence for the archetypal receptivity structures shown in figure $5(b)$ existing in principle over the considered range of wavenumbers irrespective whether the resolvent norm peaks or not and further underscores the generality of the mechanism. It would appear that our conclusion is consistent with Antkowiak (2005, figure 3.26), yet contrary to previous statements that receptivity characteristics depend on $\alpha$ (e.g. Fontane et al. 2008, p. 245). Further corroboration of insensitivity to variations in $\alpha$ will be presented in $\S 5$ when discussing resolvent-norm surfaces over $(\omega, \alpha)$ in figure 7 .

The observed selectivity in $\omega$ and $r$ but not in $\alpha$ is consistent with the matching of critical layers for all vortex models discussed in $\S 4.3$. 


\section{Robustness of linear vortex receptivity}

It is well known that a sufficiently strong jet component in the core is necessary to destabilise an isolated unstrained vortex. This fact suggests that linear stability (i.e. the discrete spectrum) quite crucially depends on the precise vortex structure. In contrast, receptivity according to definition 2.1 is rather insensitive to major changes in the reference flow as will be affirmed henceforth by comparing different vortex models. We believe that this makes sustained forcing a better candidate to explain the trailing-vortex dynamics which is found to obey universal characteristics, too.

Assessment of Lamb-Oseen, Batchelor and Moore-Saffman vortices as reference-flow models suggests the following generality conjecture which will be detailed thereafter.

CONJECTURE 5.1. Receptivity of a trailing vortex to free-stream disturbances (as defined in $\$ 2.1$ ) in the framework of $\$ 2.3$ is generic for the reference flows introduced in $\$ 2.2$.

Results for the Batchelor vortex have been computed for the case discussed in Fabre \& Jacquin (2004, p. 259) with regards to the vortex-meandering experiments of Jacquin et al. (2001) with $q=4$ and $R_{\delta}=1500$, corresponding to $R_{\Gamma}=6000 .\left(R_{\delta}:=r_{0} \delta U_{z} / v\right.$ is related to $R_{\Gamma}$ through the swirl number as $R_{\Gamma}=|q| R_{\delta}$ (Fabre \& Jacquin 2004, p. 242).) The Moore-Saffman vortex is parametrised on $n \in\{0.7,0.8\}$. While parameters in $\S 4$ were chosen for the sake of comparability to other theoretical approaches, the present choice is realistic for wind-tunnel experiments.

Contours (thin lines) of the resolvent norm in the $(\alpha, \omega)$-plane are shown in figure 7 for $m= \pm 1$ in comparison with curves of the least damped eigenvalues (thick lines). Essentially the same amplification contours and perturbation families are obtained for the Lamb-Oseen and Moore-Saffman vortex (not shown). This already suggests some generality of the perturbation dynamics with $\alpha>0, m=1$ and for $q, R_{\Gamma}$ sufficiently large.

Perturbations are conventionally classified according to their relative motion with respect to the reference flow. Thus, comparing signs of the azimuthal phase and mean velocity we define modes with $\operatorname{sgn} \omega / m \neq \operatorname{sgn} U_{\theta}$ as countergrade, modes with $\operatorname{sgn} \omega / m=$ $\operatorname{sgn} U_{\theta}$ and $|\omega / m| \in[0,1]$ as retrograde and modes with $\operatorname{sgn} \omega / m=\operatorname{sgn} U_{\theta}$ and $|\omega / m|>1$ as cograde (cf. Fabre et al. 2006, p. 241). The different regimes are indicated in figures 7 and 8. As for the Lamb-Oseen vortex, one broadly distinguishes four families of perturbation structures, namely of core $C$, viscous $V$, displacement $D$ and mixed $L$ type (see Fabre et al. 2006, pp. 247-255). Countergrade perturbations with $m=-1$ belong to the D and L1 families (Fabre et al. 2006, figure 14a) with increasing structural complexity in $r$ for subsequent branches as $\alpha$ is increased. Generally, forcing structures having $n>0$ zero crossings induce response with $n \geq 0$ roots, both being essentially localised in the core.

Different shapes of the resolvent norm $\left\|R_{m, \alpha}(\mathrm{i} \omega ; L)\right\|$ for $m= \pm 1$ are due to the axial mean-velocity breaking azimuthal symmetry (cf. $§ 2.3$ ). However, this distinction seems to be not fundamental since co-, retro- and countergrade perturbations are always associated with essentially the same families. This similarity between results for $m= \pm 1$ is further highlighted in figure 8 .

Selective non-normality can be quantified by inspection of the quantity

$$
\log _{10} \frac{\left\|R_{m, \alpha}(\mathrm{i} \omega ; L)\right\|}{d\left(\omega, \sigma_{m, \alpha}(L)\right)^{-1}} \in[0, \infty), \quad \alpha \in[0,3], \quad m= \pm 1 .
$$

By the left-hand side of (3.3) the argument is identically unity if $R_{m, \alpha}(\mathrm{i} \omega ; L)$ is (locally) effectively normal, hence, corresponding to the lower bound. As for the Lamb-Oseen 


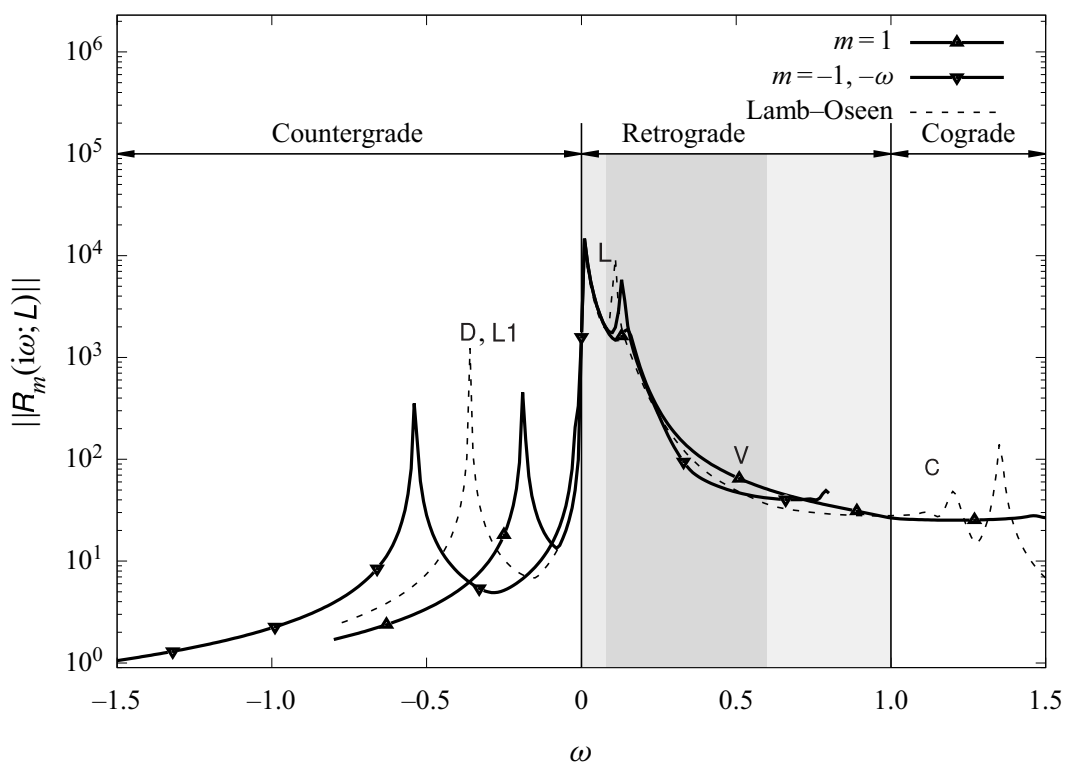

FIGURE 8. Resolvent norm of the Batchelor vortex for $R_{\Gamma}=6000$ and $q=4$ comparing $m=-1$ (plotted on the negative frequency axis $-\omega$ ) and $m=1$ for $\alpha=1.55$, as well as the resolvent norm for the Lamb-Oseen vortex $\left(R_{\Gamma}=5000\right)$. The critical-layer region of candidate receptivity is shaded in grey while the darker subset indicates the receptivity regime $R$ indicated in figure 3.

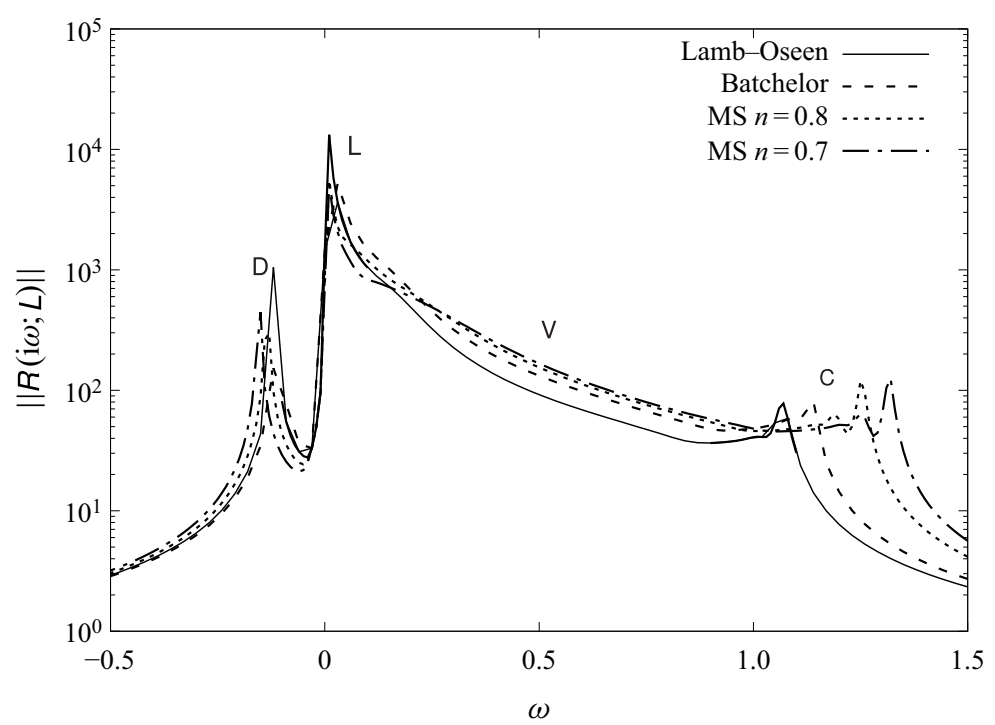

FIGURE 9. Comparison of resolvent-norm spectra for the Lamb-Oseen, Batchelor and Moore-Saffman (MS) vortices for $\alpha=0.5, m=1, R_{\Gamma}=5000, R_{\delta}=1000$ and $q=10$, showing that axial velocity has a significant effect for perturbations in the core only.

vortex, countergrade waves are always associated with an effectively normal resolvent. On the other hand, for retrograde waves, pseudo-resonance exceeds classical resonance of the equivalent normal operator by up to three orders of magnitude. Most importantly, figure 7 
confirms conjecture 4.1 that effective non-normality, and thus receptivity, are strongly confined to a small frequency band, correlated with the critical layer. These findings hold irrespective of the considered vortex model, $m= \pm 1$ and $\alpha$.

Figure 7 clearly shows that the resolvent norm of the Batchelor vortex develops sharp distinguished crests, all being aligned with the least stable eigenvalues. Each of the crests induces a dynamic regime in its neighbourhood such that the entire $(\omega, \alpha)$-plane is partitioned into different parameter subsets associated with distinguished dynamic behaviour. Therefore, the spectral signature $(\omega$ and $\alpha)$ of the forcing field will in general matter as it favours a response to different perturbation subspaces (D, L, etc.). However, as already anticipated in figure 6, grey shading in figure 7 affirms that receptivity is confined to a universal frequency band and the generic family of critical-layer waves with only small variations in alpha.

In order to gain further insight into axial-flow induced non-normality, figure 8 shows a comparison of the resolvent norm extracted from figure 7 at $\alpha=1.55$ for $m= \pm 1$ and for the Lamb-Oseen vortex. For the ease of comparison, the $m=-1$ spectrum is plotted on the negative frequency (i.e. reflected along the vertical axis at $\omega=0$ ). In all cases the central peaks of the $L$ family are universally observed as well as the general behaviour away from steady forcing. Receptivity candidates at higher frequencies are labelled $\mathrm{V}$ as in Fabre et al. (2006, p. 252) to emphasise their viscous nature. In fact, the discussion in $\S 4$ indicates that they are transitional structures between the pure critical-layer receptivity structures $L$ and core perturbations $C$. The only noticeable difference in the three cases is the location of the peaks of the D and L1 families. In the light of the above discussion this suggests that for vortices non-normal dynamics is generic while the normal dynamics is more sensitive to the reference state.

Generality and robustness is further highlighted in figure 9, showing a comparison of the resolvent norm for the three different reference states and $m=1$. The essential behaviour is unchanged by modification of the reference flow. As to be expected from discussion of the linear operator in $\S 4.1$, differences mostly manifest for core perturbations associated with the C family.

Together, figures 8 and 9 provide evidence that for vortex receptivity, and thus presumably vortex meandering, the detailed core structure of the reference state is of minor importance. These considerations support conjecture 5.1, at least for the considered dynamics, and hence a posteriori justify limitation to the canonical case of a Lamb-Oseen vortex in $\S 4$. It should be noted that this conclusion is in agreement with Antkowiak (2005, p. 47) and Pradeep \& Hussain (2006, p. 252).

Our study affirms that generally vortices are most receptive to disturbances in the critical layer. Heaton \& Peake (2007, p. 272) and Mao \& Sherwin (2012, pp. 42-44) come to the related conclusion that transient growth of the Batchelor vortex is essentially a consequence of the interaction of non-orthogonal eigenvectors pertaining to the inviscid continuous spectrum, with negligible contributions from the discrete spectrum. (Different from Antkowiak 2005, stating that combination of discrete and (unbounded) continuous spectrum.) Roy \& Subramanian (2014, p. 405) demonstrate how the inclusion of singular modes pertaining to the inviscid continuous spectrum enables interaction between vortex and free stream, suggesting that a linear model of receptivity to ambient turbulence is intimately related to the inviscid continuous spectrum.

More generally, optimal localisation of input disturbances in the critical layer is universally observed in shear flows. In the context of boundary-layer flow, analysis of the eigenmodes of the adjoint, led Hill (1995, p. 185) to conclude that perturbations are most sensitive to forcing in the critical layer. Systematic alignment of perturbations with the critical layer in boundary-layer flow similarly to our figure 5(a) is equally shown in 
McKeon (2017, figures 7, 8 and 10). The importance of critical-layer dynamics is also reported for pipe and Couette flow as well as for exact coherent states (e.g. Hall \& Sherwin 2010; McKeon \& Sharma 2010).

\section{Frequency selection in meandering experiments}

Trailing-vortex experiments reveal that meandering is principally associated with a broadband power spectral density (Devenport et al. 1996, p. 93), however, the principal energy-carrying structures are confined to a narrow frequency band (Jacquin et al. 2001; Bailey et al. 2018). Sufficiently far downstream (more than five chord lengths, say), experiments consistently report convergence towards the universal Strouhal number of $S_{m}:=f_{m} r_{1} / U_{\infty} \sim 10^{-2}$ ( $m$ stands for meandering), while closer to the wing, energy may be concentrated at a higher frequency (Bailey et al. 2018, figure 7). Hence, despite its formal broadband nature, the trailing-vortex dynamics bears spectral coherence in the sense that the most energetic feature (i.e. meandering) happens at a specific frequency irrespective of free-stream intensity, suggesting idealisation in terms of a monochromatic wave. Experiments of Bailey et al. (2018, figure 7) identify the peak frequencies $S_{m} \in$ $\{0.02,0.3\}$ for measurement stations $z \lesssim 5 c$ and $z \gtrsim 5 c$, respectively.

Direct comparison of these characteristic experimental frequencies with the spectral signature of the resolvent is, however, complicated since the two analyses do not use the same reference frames. The principal issue is a manifestation of the Doppler effect for space-time signals (Landau \& Lifshitz 1959, §68). In experiments, probing a spatio-temporally varying signal at a fixed position in space, spatial and temporal variations are indistinguishably recognised as temporal unsteadiness. Consequently, the experimentally obtained frequencies in fact correspond to the locus $D\left(S_{m}\right):=\{(\omega, \alpha) \in$ $\left.\mathbb{R}^{2} \mid S_{m}-\left(\omega+\alpha U_{\infty}\right) /(2 \pi)=0\right\}$ (up to changes in non-dimensionalisation; $r_{1} / r_{0}=1.12$ ), assuming constant background advection $U_{\infty} /\left(\Gamma_{0} /\left(2 \pi r_{0}\right)\right)=1.83$ (Bailey et al. 2018). The loci $D\left(S_{m}\right) \subset \mathbb{R}^{2}$ for $S_{m} \in\{0.02,0.3\}$ are shown in figure 7 for the case of the Batchelor vortex.

If vortex meandering is due to generalised receptivity, assessment of the effective local non-normality (shown in grey shading in figure 7) allows us to restrict considerably the range of candidate $(\omega, \alpha)$-pairs identified from the Doppler relation. Taking into account that receptivity relies on radial perturbation transport, $S_{m}=0.3$ would be composed from $1.3 \lesssim \alpha \lesssim 1.7$ and $0<\omega \lesssim 0.6$ while $S_{m}=0.02$ would have contributions from $0 \lesssim$ $\alpha \lesssim 0.1$ and $0<\omega \lesssim 0.3$. It should be noted that (nearly) steady excitation is excluded since the associated forcing-response structures are both localised in the free stream. The resolvent norm along the Doppler curve for $S_{m}=0.3$ is qualitatively identical to that shown in figure 8 . Since the resolvent norm peaks sharply at the frequency-wavenumber pair of $(\omega, \alpha) \approx(0.15,1.6)$ it is expected that the response should be dominated by this monochromatic contribution.

\section{Conclusion}

We have investigated linear vortex receptivity by means of the resolvent (i.e. a family of transfer operators mapping the external forcing to the vortex response in frequency space). Discussion of (1.1) and (1.2) makes clear that the present study is complementary to previous linear approaches. In fact, the resolvent is at the root of all linear theories. Similarity of our results with those reported in the literature is therefore of structural rather than physical origin. Nevertheless, analysis of the resolvent is best suited for the question of free-stream receptivity. Furthermore, analysis in frequency space allows us to relate the 
spectrum (determining the equivalent normal dynamics) and non-normal dynamics. Our findings confirm previous results of Heaton \& Peake (2007) and Mao \& Sherwin (2012) in time domain (analysis of the propagator $T(t)$ ).

The essential prerequisite for linear receptivity is non-normality. Formulation of the problem in terms of the resolvent is appropriate to quantify non-normality locally (in frequency space). Despite the governing operator being non-normal, it was shown that the effective behaviour for vortices depends on the excitation frequency (called selective non-normality). To the best of our knowledge, this work contains the first systematic use of resolvent bounds to classify non-normality of the linear vortex dynamics (considerable use of these bounds can be found in e.g. Reddy, Schmid \& Henningson (1993), too).

We found non-normality of linear vortex dynamics to be essentially restricted to frequencies aligned with the inviscid continuous spectrum (characterising a perturbation dynamics with stationary material advection). Accordingly, the associated forcing structures are localised in the critical layer. Using an upper bound of non-normality in terms of the numerical range, we showed that disturbance alignment with the critical layer is necessary to maximise non-normality. This finding is given physical meaning by consideration of the instantaneous variation of the integral kinetic energy.

Considering Lamb-Oseen, Batchelor and Moore-Saffman vortices as reference states, we found the above features of non-normality to be robust. In particular, irrespective of the vortex model, linear dynamics turns out to be frequency-selectively non-normal on the same range with forcing structures being systematically localised about the critical layer. On the contrary, non-normality is almost invariant under variations of the axial wavenumber in the considered range. We conclude that free-stream receptivity as a consequence of non-normal dynamics is largely independent of the precise vortex-core structure and in particular does not rely on axial mean velocity. This is in stark contrast to results of asymptotic stability (discrete spectrum) which crucially depends on the vortex model. Yet, universality of experimental trailing-vortex dynamics suggests that it should be associated with a robust mechanism, common for a large class of vortices. As motivated in $\S 1$, we suggest a model of trailing-vortex dynamics as a linear system driven by the advective nonlinearity.

\section{Acknowledgements}

This work has been supported by the French Ministry of Civil Aviation (DGAC) under PHYWAKE (PHYsics of WAKE vortices) research program. We are grateful to the anonymous referees for their exceptional investment and insightful suggestions.

\section{Declaration of interests}

The authors report no conflict of interest.

\section{Appendix. Numerical implementation, verification and validation}

The present study relies on canonical and spectral decomposition of the linear operators $R(s ; L), L$ and $L^{\dagger}$ as given in (2.3) which are approximated using a finite-element discretisation (see $\S 2$ of Sipp \& Marquet 2013 for details). The implementation uses freefem++ (Hecht 2018) which uses the ARPACK library to solve eigenvalue problems (Lehoucq, Sorensen \& Yang 1997). Finite-element functions are fixed to the linear P1 and quadratic P2 Lagrangian finite elements for pressure and velocity, respectively (Hecht 2018, pp. 174-175). The radial domain in all computations is $R=\left(0, r_{\max }\right)=$ 
$(0,1] \cup(1,6] \cup\left(6, r_{\max }\right)=R_{1} \cup R_{2} \cup R_{3}$ with $r_{\text {max }}=30$. The number of elements in each part of this partition is 200, 200 and 150 for $R_{1}, R_{2}$ and $R_{3}$, respectively. Grid refinement studies have been done for verification and convergence of the presented results is assured by variation of the maximum radius $r_{\max } \in[30,200]$.

The results of the canonical decomposition of the resolvent have been validated against the studies of Guo \& Sun (2011) and Blanco-Rodríguez et al. (2017). Spectral decomposition of $L$ is validated by comparing with the results of Fabre et al. (2006). To the best of our knowledge spectral decomposition of the adjoint $L^{\dagger}$ has not been reported for vortices before; validation of the computation is therefore obtained by assuring condition (i) $\sigma(L)=\overline{\sigma\left(L^{\dagger}\right)}$ on the spectra and (ii) bi-orthogonality to hold (Friedman 1962, p. 112; Kato 1980, theorem 6.22).

\section{REFERENCES}

ABID, M. 2008 Nonlinear mode selection in a model of trailing line vortices. J. Fluid Mech. 605, 19-45.

AntKowiak, A. 2005 Dynamique aux temps courts d'un tourbillon isolé. PhD thesis, Université Paul Sabatier de Toulouse.

Antkowiak, A. \& Brancher, P. 2004 Transient energy growth for the Lamb-Oseen vortex. Phys. Fluids 16 (1), L1-L4.

ARNOL'D, V. I. 1992 Ordinary Differential Equations. Springer.

Ash, R. L. \& Khorrami, M. R. 1995 Vortex stability. In Fluid Vortices (ed. S. I. Green), pp. 317-372. Springer.

Bailey, S. C. C., Pentelow, S., Ghimire, H. C., Estejab, B., Green, M. A. \& Tavoularis, S. 2018 Experimental investigation of the scaling of vortex wandering in turbulent surroundings. J. Fluid Mech. 843, 722-747.

BAKer, G. R., BARKer, S. J., BOFAH, K. K. \& SAFFMAN, P. G. 1974 Laser anemometer measurements of trailing vortices in water. J. Fluid Mech. 65, 325-336.

BANDYOPADHYAY, P. R., STEAD, D. J. \& ASH, R. L. 1991 Organized nature of a turbulent trailing vortex. AIAA J. 29 (10), 1627-1633.

BAtChELOR, G. K. 1964 Axial flow in trailing line vortices. J. Fluid Mech. 20, 645-658.

Blanco-Rodríguez, F. J., Rodríguez-García, J. O., Parras, L. \& del Pino, C. 2017 Optimal response of Batchelor vortex. Phys. Fluids 29 (6), 064108.

Devenport, W. J., Rife, M. C., Liapis, S. I. \& Follin, G. J. 1996 The structure and development of a wing-tip vortex. J. Fluid Mech. 312, 67-106.

ENGEL, K.-J. \& NAGEL, R. 2000 One-Parameter Semigroups for Linear Evolution Equations. Springer.

FABRE, D. \& JACQUIN, L. 2004 Viscous instabilities in trailing vortices at large swirl numbers. J. Fluid Mech. 500, 239-262.

FABRE, D., SipP, D. \& JACQUin, L. 2006 Kelvin waves and the singular modes of the Lamb-Oseen vortex. J. Fluid Mech. 551, 235-274.

FARrell, B. F. \& IoAnnou, P. J. 1996 Generalized stability theory. Part I: autonomous operators. J. Atmos. Sci. 53 (14), 2025-2040.

Feys, J. \& MASLOWE, S. A. 2014 Linear stability of the Moore-Saffman model for a trailing wingtip vortex. Phys. Fluids 26 (2), 024108.

Fontane, J., Brancher, P. \& FABRe, D. 2008 Stochastic forcing of the Lamb-Oseen vortex. J. Fluid Mech. 613, 233-254.

Friedman, B. 1962 Principles and Techniques of Applied Mathematics. Courier Dover Publications.

García-Ortiz, J. H., Dominguez-Vazquez, A., Serrano-Aguilera, J. J., Parras, L. \& DEL PINO, C. 2019 A complementary numerical and experimental study of the influence of Reynolds number on theoretical models for wingtip vortices. Comput. Fluids 180, 176-189.

Guo, Z.-W., Chen, C. \& SUN, D.-J. 2011 Stochastic forcing of the Batchelor vortex. In AIP Conference Proceedings, vol. 1376, pp. 302-304. AIP.

Guo, Z.-W. \& Sun, D.-J. 2011 Optimal response in the Lamb-Oseen vortex. Phys. Lett. A 375 (36), 3191-3195. 
Gustafson, K. E. \& RaO, D. K. M. 1997 Numerical Range. The Field of Values of Linear Operators and Matrices. Springer.

HALL, P. \& SHERWIN, S. 2010 Streamwise vortices in shear flows: harbingers of transition and the skeleton of coherent structures. J. Fluid Mech. 661, 178-205.

Haller, G., Hadjighasem, A., Farazmand, M. \& Huhn, F. 2016 Defining coherent vortices objectively from the vorticity. J. Fluid Mech. 795, 136-173.

Heaton, C. J., Nichols, J. W. \& Schmid, P. J. 2009 Global linear stability of the non-parallel Batchelor vortex. J. Fluid Mech. 629, 139-160.

Heaton, C. J. \& Peake, N. 2007 Transient growth in vortices with axial flow. J. Fluid Mech. 587, 271-301.

Hecht, F. 2018 Freefem++. Version 3.56-2, 3rd edn. Laboratoire Jacques-Louis Lions, Université Pierre et Marie Curie.

HILl, D. C. 1995 Adjoint systems and their role in the receptivity problem for boundary layers. J. Fluid Mech. 292, 183-204.

IUNGO, G. V. 2017 Wandering of a wing-tip vortex: rapid scanning and correction of fixed-point measurements. J. Aircraft 54 (5), 1779-1790.

JACOBS, R. G. \& DURBin, P. A. 1998 Shear sheltering and the continuous spectrum of the Orr-Sommerfeld equation. Phys. Fluids 10 (8), 2006-2011.

JaCQuin, L., FABRe, D., GefFroy, P. \& Coustols, E. 2001 The properties of a transport aircraft wake in the extended near field: an experimental study. In 39th AIAA Aerospace Sciences Meeting and Exhibit, AIAA Paper, 2001-1038.

JOSEPH, D. D. 1976 Stability of Fluid Motions I. Springer.

Karami, M., Hangan, H., Carassale, L. \& Peerhossaini, H. 2019 Coherent structures in tornado-like vortices. Phys. Fluids 31 (8), 085118.

Kato, T. 1980 Perturbation Theory for Linear Operators. Springer.

Kato, T. \& Fujita, H. 1962 On the nonstationary Navier-Stokes system. Rend. Seminario Mat. Univ. Padova 32, 243-260.

LANDAHL, M. T. 1967 A wave-guide model for turbulent shear flow. J. Fluid Mech. 29, 441-459.

LANDAU, L. D. \& Lifshitz, E. M. 1959 Fluid Mechanics. Pergamon Press.

LE Dizès, S. 2004 Viscous critical-layer analysis of vortex normal modes. Stud. Appl. Maths 112 (4), $315-332$.

LehoucQ, R. B., Sorensen, D. C. \& YAng, C. 1997 Arpack Users Guide: Solution of Large Scale Eigenvalue Problems by Implicitly Restarted Arnoldi Methods. SIAM.

LEIBOVICH, S. \& STEWARTSON, K. 1983 A sufficient condition for the instability of columnar vortices. J. Fluid Mech. 126, 335-356.

MAO, X. \& SHERWIN, S. 2011 Continuous spectra of the Batchelor vortex. J. Fluid Mech. 681, 1-23.

MAO, X. \& Sherwin, S. 2012 Transient growth associated with continuous spectra of the Batchelor vortex. J. Fluid Mech. 697, 35-59.

Marshall, J. S. \& Beninati, M. L. 2005 External turbulence interaction with a columnar vortex. J. Fluid Mech. 540, 221-245.

McKeon, B. J. 2017 The engine behind (wall) turbulence: perspectives on scale interactions. J. Fluid Mech. 817, 1-86.

McKeon, B. J. \& Sharma, A. S. 2010 A critical-layer framework for turbulent pipe flow. J. Fluid Mech. 658, 336-382.

Melander, M. V. \& HUSSAIN, F. 1993 Coupling between a coherent structure and fine-scale turbulence. Phys. Rev. E 48 (4), 2669-2689.

Moore, D. W. \& Saffman, P. G. 1973 Axial flow in laminar trailing vortices. Proc. R. Soc. Lond. A 333 (1595), 491-508.

Morkovin, M. V. 1988 Recent Insights into Instability and Transition to Turbulence in Open-Flow Systems. Institute for Computer Applications in Science and Engineering, NASA Langley Research Center.

PradeEP, D. S. \& Hussain, F. 2006 Transient growth of perturbations in a vortex column. J. Fluid Mech. $550,251-288$. 
Reddy, S. C., Schmid, P. J. \& Henningson, D. S. 1993 Pseudospectra of the Orr-Sommerfeld operator. SIAM J. Appl. Maths 53 (1), 15-47.

Reddy, S. C. \& TREfethen, L. N. 1994 Pseudospectra of the convection-diffusion operator. SIAM J. Appl. Maths 54 (6), 1634-1649.

Riesz, F. \& SZ.-NAGY, B. 1956 Functional Analysis. Blackie.

RoY, A. \& Subramanian, G. 2014 Linearized oscillations of a vortex column: the singular eigenfunctions. J. Fluid Mech. 741, 404-460.

SARIC, W. S., REeD, H. L. \& Kerschen, E. J. 2002 Boundary-layer receptivity to freestream disturbances. Annu. Rev. Fluid Mech. 34 (1), 291-319.

Sharma, A. S., Mezić, I. \& McKeon, B. J. 2016 Correspondence between Koopman mode decomposition, resolvent mode decomposition, and invariant solutions of the Navier-Stokes equations. Phys. Rev. Fluids 1, 032402.

SIPP, D. \& MARQUET, O. 2013 Characterization of noise amplifiers with global singular modes: the case of the leading-edge flat-plate boundary layer. Theor. Comput. Fluid Dyn. 27 (5), 617-635.

Sipp, D., Marquet, O., Meliga, P. \& Barbagallo, A. 2010 Dynamics and control of global instabilities in open-flows: a linearized approach. Appl. Mech. Rev. 63 (3), 1-26.

SoHr, H. 2001 The Navier-Stokes equations: An Elementary Functional Analytic Approach. Springer.

TAKAHASHI, N., ISHII, H. \& MiYAZAKI, T. 2005 The influence of turbulence on a columnar vortex. Phys. Fluids 17 (3), 035105.

Ting, L., Klein, R. \& KNiO, O. M. 2007 Vortex Dominated Flows: Analysis and Computation for Multiple Scale Phenomena. Springer Science \& Business Media.

Towne, A., SCHMidT, O. T. \& Colonius, T. 2018 Spectral proper orthogonal decomposition and its relationship to dynamic mode decomposition and resolvent analysis. J. Fluid Mech. 847, 821-867.

Trefethen, L. N. \& Embree, M. 2005 Spectra and Pseudospectra: The Behavior of Nonnormal Matrices and Operators. Princeton University Press.

Van JaArsveld, J. P. J., Holten, A. P. C., Elsenaar, A., Trieling, R. R. \& Van Heijst, G. J. F. 2011 An experimental study of the effect of external turbulence on the decay of a single vortex and a vortex pair. J. Fluid Mech. 670, 214-239.

Viola, F., Arratia, C. \& Gallaire, F. 2016 Mode selection in trailing vortices: harmonic response of the non-parallel Batchelor vortex. J. Fluid Mech. 790, 523-552.

WANG, Z. \& GURSUL, I. 2012 Unsteady characteristics of inlet vortices. Exp. Fluids 53 (4), 1015-1032. 